\title{
DEMANDAS DE ENERGÍA HISTERÉTICA EN OSCILADORES ELASTOPLÁSTICOS SUJETOS A RUIDO BLANCO GAUSSIANO
}

\author{
Danny Arroyo ${ }^{(1)}$ y Mario Ordaz ${ }^{(2)}$
}

\begin{abstract}
RESUMEN
Se presenta una solución teórica para estimar la energía histerética por unidad de masa disipada por un oscilador elastoplástico sujeto a una familia de ruidos blancos gaussianos. La solución fue obtenida con base en el modelo propuesto originalmente por Karnopp y Scharton. Con el método que se propone, los resultados sólo dependen del periodo y de la resistencia del oscilador, del nivel de amortiguamiento y de la densidad espectral de potencia de la familia de ruidos blancos. Las expresiones propuestas son de carácter más general que las expresiones de Karnopp y Scharton y, a diferencia de estas últimas, son aplicables a sistemas con propiedades comúnmente utilizadas en ingeniería sísmica.
\end{abstract}

\begin{abstract}
A theoretical method to compute the hysteretic energy demand for elastoplastic oscillators subjected to Gaussian white noise excitation is presented. The solution presented was obtained from the equivalent model proposed by Karnopp y Scharton. With the solution presented, results depend only on the period and the yield strength of the oscillator, its level of damping and the spectral density function of the white noise. The solution proposed is useful to estimate the hysteretic energy demands for systems with properties commonly found in earthquake engineering. Also, the ability of the solution presented to compute the hysteretic energy demands for elastoplastic oscillators subjected to wide band earthquake ground motions is shown.
\end{abstract}

\section{INTRODUCCIÓN}

La normatividad actual de diseño sísmico, a nivel mundial, utiliza el desplazamiento máximo que una estructura experimenta durante un evento sísmico como indicador de su comportamiento estructural. Los reglamentos de diseño establecen que si el desplazamiento máximo que presenta una estructura durante un evento sísmico es menor que su desplazamiento último $\left(x_{u}\right)$ ante carga monotónica la estructura tendrá un nivel de seguridad adecuado contra colapso.

Artículo recibido el 17 de mayo del 2005 y aprobado para su publicación el 17 de marzo del 2006. Se aceptarán comentarios y/o discusiones hasta cinco meses después de su publicación.

(1) Universidad Autónoma Metropolitana, Departamento de Materiales. Av. San Pablo \# 180. Col. Reynosa, 02200 México, DF. aresda@,correo.azc.uam.mx

(2) Instituto de Ingeniería, UNAM,Ciudad Universitaria, 04510 México, DF. mors@pumas.iingen.unam.mx 
Sin embargo, algunos investigadores han mostrado que la respuesta máxima de desplazamiento, por si sola, no siempre es un buen indicador del comportamiento estructural, sobre todo ante eventos sísmicos de larga duración (Iemura 1980, Park y Ang 1985, Fajfar 1992, Terán 1996, Terán y Jirsa 2003). Debido a que las propiedades mecánicas de una estructura, cuando se sujeta a una excitación sísmica, se pueden deteriorar en cada ciclo de carga lo que puede conducir a la falla estructural a un nivel de desplazamiento menor que la capacidad máxima de desplazamiento ante carga monotónica. Este fenómeno también se conoce como fatiga de bajo número de ciclos.

El nivel de degradación depende de las propiedades mecánicas de los elementos así como de la amplitud, secuencia y número de ciclos de carga, por lo que es complicado establecer un método racional de diseño.

Una forma de considerar en el diseño la degradación de las propiedades de una estructura debido a los ciclos de carga que una excitación sísmica le impone es el uso de índices de daño.

Un índice de daño es una función matemática que relaciona las demandas que experimenta un sistema con sus capacidades, con el fin de establecer el nivel de deterioro de dicho sistema cuando se le sujeta a determinada historia de carga. Diferentes investigadores han estudiado la aplicación de índices de daño para el diseño sísmico (Fajfar 1992, Bertero y Bertero 1992, Cosenza y Manfredi 1996, Arroyo y Terán 2002 y Ridell y García 2002).

En general, los índices de daño están calibrados adecuadamente para elementos estructurales; normalmente un valor del índice de daño igual a la unidad se asocia a la falla estructural. Cabe mencionar que no existe un consenso de lo que constituye la falla de un elemento. En los diferentes índices de daño disponibles la falla se ha definido de acuerdo al criterio de cada investigador.

Las demandas consideradas generalmente por los diferentes índices de daño son el desplazamiento máximo y las demandas de desplazamiento acumulado, caracterizadas mediante la demanda de energía histerética disipada, mientras que las características mecánicas consideradas son la capacidad resistente, la capacidad de desplazamiento y la estabilidad del ciclo histerético. Existen numerosas formulaciones de índices de daño; una recopilación y descripción detallada de los más utilizados puede encontrarse en Williams y Sexsmith (1995) y en Mehanny y Deirlein (2000).

El índice mas utilizado es el propuesto por Park y Ang (1985), $I D_{P A}$, definido en la ecuación (1), debido a que existe numerosa evidencia experimental que lo sustenta, principalmente en elementos de concreto reforzado.

$$
I D_{P A}=\frac{x_{\max }}{x_{u}}+\beta \frac{E_{H}}{F_{y} x_{u}}
$$


donde $x_{\max }$ es la demanda de desplazamiento máximo, $E_{H}$ es la energía histerética disipada, $x_{u}$ es el desplazamiento último ante carga monotónica, $F_{y}$ es la resistencia de fluencia del sistema y $\beta$ es un parámetro relacionado con la estabilidad del ciclo histerético.

El $I D_{P A}$ representa una solución aproximada al problema, ya que como puede observarse, no contempla ni la amplitud ni la secuencia en la que se presentan los ciclos de carga y propone una combinación lineal entre el daño producido por las demandas máximas y las demandas acumuladas. Diferentes investigadores han mostrado las inconsistencias que tiene el $I D_{P A}$ (Williams y Sexsmith 1995, Terán 1996, Mehanny y Deirlein 2000, Bozorgnia y Bertero 2003).

Cabe mencionar que evaluar los diferentes índices de daño disponibles en la literatura está fuera del alcance de este trabajo. El $I D_{P A}$ se presenta en esta sección sólo a manera de referencia y para ilustrar su aplicación en el contexto del diseño sísmico de estructuras.

De acuerdo con la ecuación (1) la capacidad de desplazamiento que un sistema requiere para evitar la falla es:

$$
x_{u}=x_{\max }+\beta \frac{E_{H}}{F_{y}}
$$

De acuerdo con la ecuación (2), para determinar la capacidad de deformación que debe suministrarse a un sistema para evitar su falla es necesario conocer la demanda de desplazamiento máximo, la demanda de energía histerética disipada, la estabilidad del ciclo histerético (caracterizada a través de $\beta$ ) y la resistencia de fluencia del sistema.

Otro enfoque de la aplicación del $I D_{P A}$ en el contexto del diseño sísmico se muestra en la ecuación (3).

$$
x_{\max }=x_{u}-\beta \frac{E_{H}}{F_{y}}
$$

De acuerdo a la ecuación (3) la demanda máxima de desplazamiento que un sistema puede experimentar cuando se le sujeta a una excitación sísmica, sin alcanzar la falla, depende de su capacidad de desplazamiento, de su resistencia de fluencia, de la estabilidad de su ciclo histerético y de la demanda de energía histerética disipada.

Independientemente del enfoque considerado para aplicar el $I D_{P A}$, y otros índices de daño, en el contexto del diseño sísmico de estructuras es necesario contar con estimaciones confiables de las demandas sísmicas y de las capacidades estructurales.

Diferentes investigadores han propuesto expresiones para estimar dichas demandas basadas en estudios estadísticos (Fajfar y Vidic 1992 y Manfredi 2001). Sin embargo, pocos esfuerzos se han encaminado a obtener expresiones teóricas (Karnopp y Scharton 1966). 
Las expresiones obtenidas de estudios estadísticos son herramientas muy importantes para el diseño sísmico de estructuras. Pero debe reconocerse que los resultados obtenidos están influidos por las características y el número de acelerogramas incluidos en la muestra utilizada en los análisis de regresión y que la aplicabilidad de las expresiones está limitada a los intervalos de los diferentes parámetros considerados en los análisis.

En este estudio se presenta una solución teórica para estimar la energía histerética disipada por un oscilador elastoplástico sujeto a ruido blanco gaussiano. Además, se muestra que el método puede ser aplicado para estimar la demanda de energía histerética disipada por osciladores elastoplásticos sujetos a movimientos sísmicos de banda ancha.

\section{EL MODELO DE KARNOPP Y SCHARTON}

Considere el oscilador elastoplástico con masa $(m)$, constante de amortiguamiento $(c)$, rigidez inicial $(k)$, resistencia de fluencia $F_{y}$ desplazamiento inicial de fluencia $X_{f}$, mostrado en la figura 1 .

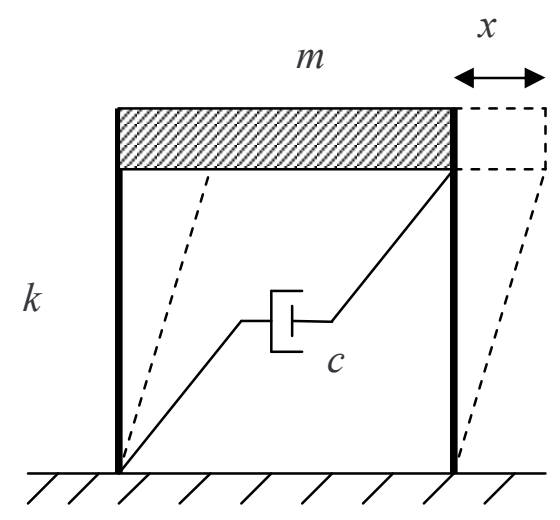

Figura 1. Oscilador amortiguado

Dicho sistema se somete a una familia de aceleraciones formada por ruidos blancos gaussianos. Esta familia está definida por su densidad espectral de potencia $(S(\omega)$ dada por la ecuación (4).

$$
S(\omega)=\lim _{t_{d} \rightarrow \infty} \frac{E\left[|F(\omega)|^{2}\right]}{2 t_{d}}
$$

donde $t_{d}$ es la duración de la señal, $|F(\omega)|$ es la amplitud del espectro de Fourier de la señal y E[ ] es un operador que denota valor esperado. Para el caso de ruido blanco gaussiano la función $S(\omega)$ es una constante con valor igual a $S_{0}$.

Cuando se le sujeta a la aceleración considerada, el sistema cambia varias veces del intervalo elástico al inelástico y viceversa, por lo que es necesario llevar un registro de la deformación plástica permanente para establecer el nuevo desplazamiento de fluencia después de 
cada excursión plástica. Esto dificulta encontrar una solución analítica para estimar la respuesta de este tipo de sistemas. Karnopp y Scharton (1966) propusieron un modelo que evita el tener que llevar un registro de la deformación permanente después de cada excursión plástica del sistema.

El modelo consiste en construir un proceso imaginario $(\eta(t))$ eliminando todos los instantes en que el sistema se encuentre en el intervalo inelástico de comportamiento y uniendo los segmentos de respuesta lineal restantes.

El proceso resultante es equivalente a colocar unas barreras infinitamente rígidas y perfectamente elásticas en un desplazamiento igual al desplazamiento de fluencia inicial del sistema. El proceso resultante se ilustra en la figura 2.

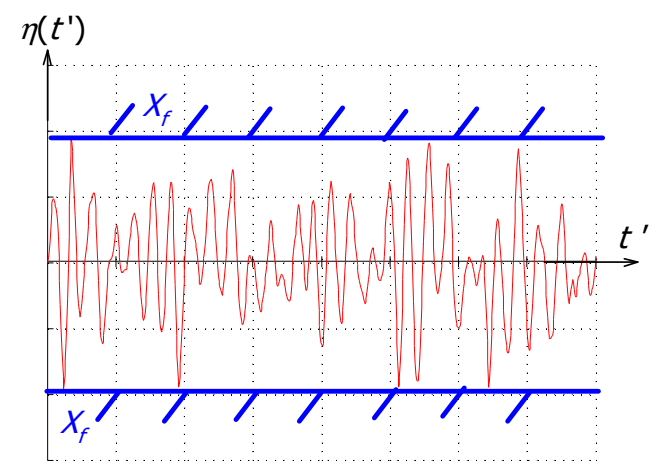

Figura 2. Modelo de Karnopp y Scharton

Dado que el sistema en el proceso $\eta(t)$ se comporta linealmente, el sistema queda descrito por la ecuación (5), suponiendo que el desplazamiento permanente es cero.

$$
\eta+2 \xi \omega_{0} \eta+\omega_{0}^{2} \eta=-a(t)
$$

Siempre que $|\eta(t)|<X_{f}$

Mientras el oscilador permanezca elástico el valor esperado del desplazamiento y de la velocidad están definidos por (Karnopp y Scharton 1966):

$$
\begin{aligned}
& E[\eta(t)]=\eta_{0} g(t)+\eta_{0} h(t) \\
& E[\eta(t)]=\eta_{0} g(t)+\eta_{0} \dot{h}(t)
\end{aligned}
$$

donde $\omega_{0}$ es la frecuencia de vibración, $\eta_{0}$ y $\eta_{0}$ son el desplazamiento y la velocidad iniciales respectivamente y

$$
g(t)=e^{-\xi \omega_{0} t}\left(\frac{\xi}{\sqrt{1-\xi^{2}}} \sin \left(\omega_{d} t\right)+\cos \left(\omega_{d} t\right)\right)
$$




$$
\begin{array}{r}
h(t)=\frac{e^{-\xi \omega_{0} t}}{\omega_{d}} \sin \left(\omega_{d} t\right) \\
\omega_{d}=\omega_{0} \sqrt{1-\xi^{2}}
\end{array}
$$

Además, la varianza del desplazamiento $\left(\sigma_{\eta t}^{2}\right)$, la varianza de la velocidad $\left(\sigma_{\eta t}^{2}\right)$ y el coeficiente de correlación entre desplazamiento y velocidad $\left(\rho_{t}\right)$ quedan definidos por (Karnopp y Scharton 1966):

$$
\begin{aligned}
& \sigma_{\eta t}^{2}=\sigma_{\eta}^{2}+\frac{\sigma_{\eta}^{2}}{\omega_{d}^{2}} e^{-2 \xi \omega_{0} t}\left(-\omega_{0}^{2}+\xi^{2} \omega_{0}^{2} \cos \left(2 \omega_{d} t\right)-\xi \omega_{0} \omega_{d} \sin \left(2 \omega_{d} t\right)\right) \\
& \sigma_{\eta t}^{2}=\sigma_{\eta}^{2}+\frac{\sigma_{\eta}^{2}}{\omega_{d}^{2}} e^{-2 \xi \omega_{0} t}\left(-\omega_{0}^{2}+\xi^{2} \omega_{0}^{2} \cos \left(2 \omega_{d} t\right)+\xi \omega_{0} \omega_{d} \sin \left(2 \omega_{d} t\right)\right) \\
& \rho_{t}=\frac{S_{0} h^{2}(t)}{\sigma_{\eta t} \sigma_{\eta t}}
\end{aligned}
$$

donde:

$$
\begin{gathered}
\sigma_{\eta}^{2}=\frac{\pi S_{0}}{2 \xi \omega_{0}^{3}} \\
\sigma_{\eta}^{2}=\frac{\pi S_{0}}{2 \xi \omega_{0}}
\end{gathered}
$$

En el sistema original la energía cinética al inicio de cada fluencia se disipa durante la excursión plástica, mientras que en el proceso $\eta(t)$ la disipación ocurre de forma instantánea al contacto con las barreras.

Como la aceleración es un ruido blanco gaussiano, al eliminar los intervalos de tiempo en que el oscilador fluye no se modifican las propiedades estadísticas de la aceleración pues su autocorrelación es igual a cero para cualquier intervalo finito de tiempo.

El proceso $\eta(t)$ no presenta acumulación del desplazamiento permanente como en el sistema original pero el valor esperado del desplazamiento plástico $\left(\Delta x_{p}\right)$ que ocurre cada vez que el oscilador fluye se estima conforme a lo siguiente:

Considere el intervalo de tiempo definido por el instante en que el oscilador toca alguna barrera (comienza a fluir) y el instante en que oscilador regresa de nuevo dentro de las barreras (descarga). 
Durante dicho intervalo el comportamiento del oscilador queda definido por la ecuación (16).

$$
\eta+2 \xi \omega_{0} \eta+\operatorname{sign}(\eta) \frac{F_{y}}{m}=-a(t)
$$

Se puede demostrar que los valores esperados de las energías por unidad de masa, cinética $\left(E_{K}\right)$, viscosa $\left(E_{H \xi}\right)$, plástica $\left(E_{H \mu}\right)$ y de entrada $\left(E_{I}\right)$, para el intervalo de tiempo considerado son, respectivamente:

$$
\begin{aligned}
& E\left[E_{K}\right]=\frac{1}{2} E\left[v_{f}^{2}\right] \\
& E\left[E_{H \xi}\right]=2 \xi \omega_{0} \int_{t_{1}}^{t_{2}} E\left[\eta^{2}\right] d t \\
& E\left[E_{H_{\mu}}\right]=\frac{F_{y}}{m} E\left[\left|\Delta x_{p}\right|\right] \\
& E\left[E_{I}\right]=-\int_{t_{1}}^{t_{2}} E[a(t) \eta] d t
\end{aligned}
$$

donde $v_{f}$ es la velocidad del oscilador al contacto con la barrera, $\Delta x_{p}$ es igual a la diferencia entre el desplazamiento en la descarga y el desplazamiento al inicio de la fluencia y $t_{1}$ y $t_{2}$ son el tiempo en que inicia y termina la fluencia, respectivamente.

Para ruido blanco gaussiano $E[a(t)]=0$, por lo que el valor esperado de la energía de entrada es cero. Además, para sistemas con poco amortiguamiento, como los que se estudian en ingeniería estructural, es razonable despreciar el término de la energía viscosa.

Con base en las observaciones anteriores se concluye que, en promedio, la energía cinética que tiene el oscilador cuando toca alguna barrera se disipa en energía plástica durante la fluencia del sistema.

Además, se puede demostrar que, bajo la suposición anterior, $E\left[v_{f}^{2}\right]$ y $E\left[\left|\Delta x_{p}\right|\right]$ están relacionados de la siguiente manera:

$$
E\left[\left|\Delta x_{p}\right|\right]=\frac{E\left[v_{f}^{2}\right]}{2 \omega_{0}^{2} X_{f}}
$$

Entonces, para calcular la energía plástica disipada por el oscilador sólo es necesario conocer el valor de $E\left[v_{f}^{2}\right]$ y el número de veces que se toca la barrera durante el proceso. 
Karnopp y Scharton propusieron una solución aproximada para estimar el valor de $E\left[\left|\Delta x_{p}\right|\right]$ basados en la suposición de que el oscilador esta regresando de una fluencia y que el tiempo que transcurre para que ocurra el siguiente contacto con las barreras es igual a:

$$
t_{f}=\frac{\pi}{\omega_{d}}
$$

Las hipótesis de Karnopp y Scharton se ilustran en la figura 3.

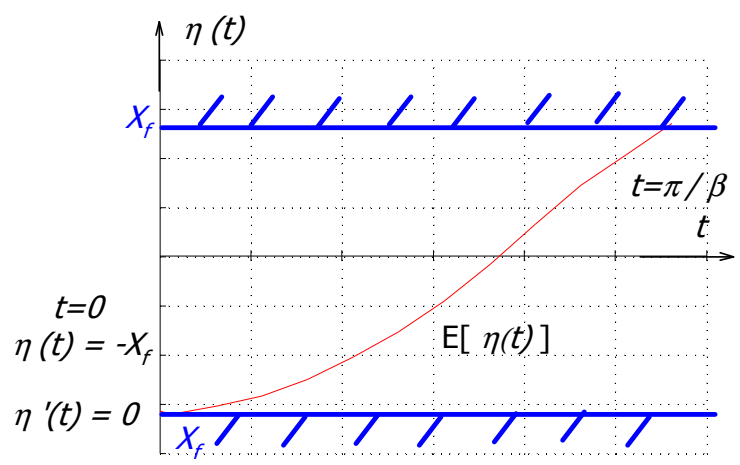

Figura 3. Hipótesis de Karnopp y Scharton para la solución del oscilador considerado

La expresión propuesta por Karnopp y Scharton para estimar el valor esperado del desplazamiento plástico durante las fluencias del oscilador es:

$$
E\left[\left|\Delta x_{p}\right|\right]=\frac{\sigma_{\eta t}}{P_{0} \sqrt{2 \pi}} e^{\left(\frac{-a^{2} \delta}{8}\right)}-\frac{a \sqrt{\delta} \sigma_{\eta t}}{2}
$$

Donde:

$$
\begin{aligned}
& \delta=\frac{2 \pi \xi}{\sqrt{1-\xi^{2}}} \\
& a=\frac{x_{f}}{\sigma_{\eta}} \\
& P_{0}=1-\Phi\left(\frac{a \sqrt{\delta}}{2} \sigma_{\eta t}\right)=1-\Phi\left(\frac{a\left(e^{-\delta / 2}-1\right)}{\sqrt{1-e^{-\delta}}}\right)
\end{aligned}
$$

$\Phi()$ es un operador que denota distribución normal estándar, $\sigma_{\eta t}$ es la desviación estándar del desplazamiento elástico del oscilador evaluada para $t=t_{f}$, y $\sigma_{\eta}$ es la desviación estándar estacionaria del desplazamiento definida por la ecuación (14). 
La tasa de contactos en la barrera por unidad de tiempo es (Karnopp y Scharton 1966):

$$
v_{c}=a \frac{\sqrt{\xi}}{\sqrt{2 \pi}} \omega_{0} e^{-\frac{a^{2}}{2}}\left(\frac{1}{1-2 P_{0}+P_{0}}\right)
$$

El procedimiento anterior es aplicable a sistemas con valores de $a$ pequeños. Para sistemas con valores de $a$ grandes (mayores a la unidad) el tiempo que se requiere para que el oscilador toque la barrera es grande respecto a su periodo y es aplicable la solución estacionaria (Karnopp y Scharton 1966).

Para el caso estacionario, el valor esperado del cuadrado de la velocidad cuando se toca la barrera es:

$$
E\left[v_{f}^{2}\right]_{e s t}=\frac{\pi S_{0}}{2 \xi \omega_{0}}
$$

La tasa de contactos con la barrera para el caso estacionario también fue definida por Karnopp y Scharton (1966):

$$
v_{e s t}=\frac{\sigma_{\eta}}{\pi \sigma_{\eta}} e^{-\frac{X_{f}^{2}}{2 \sigma_{\eta}^{2}}}
$$

Vanmarcke y Lai (1967) observaron que la solución de Karnopp y Scharton es válida sólo cuando el proceso se vuelve casi estacionario, lo cual ocurre cuando el nivel de resistencia es alto y pasa un tiempo considerable para que se presente el primer contacto con las barreras.

La principal debilidad de la solución de Karnopp y Scharton es que todos los sistemas con el mismo periodo de vibración tienden a tocar la barrera al mismo tiempo, independientemente de su resistencia. En realidad, mientras menor sea la resistencia, las barreras se encontrarán más cercanas y el sistema las tocará más rápido.

Está debilidad no es importante para los sistemas para los que fue desarrollado su planteamiento, pues tienen altas resistencias y altas frecuencias de vibración (del orden de 1600 $\mathrm{rad} / \mathrm{s})$.

Sin embargo, para los sistemas que comúnmente aparecen en la ingeniería estructural esta debilidad, como se muestra mas adelante, se refleja en errores muy importantes en la estimación de las demandas de energía histerética. 


\section{SOLUCIÓN PROPUESTA}

\section{Aspectos preliminares.}

La figura 4 muestra las cinco posibles trayectorias que el oscilador sujeto a ruido blanco puede seguir partiendo de las condiciones iniciales $\eta_{0}=-X_{f} y \eta_{0}^{\prime}=0$.

a) El oscilador parte con velocidad positiva y toca la barrera positiva

b) El oscilador parte con velocidad positiva y toca la barrera negativa

c) El oscilador parte con velocidad positiva y no toca ninguna barrera

d) El oscilador parte con velocidad negativa y toca la barrera negativa

e) El oscilador parte con velocidad negativa y no toca ninguna barrera

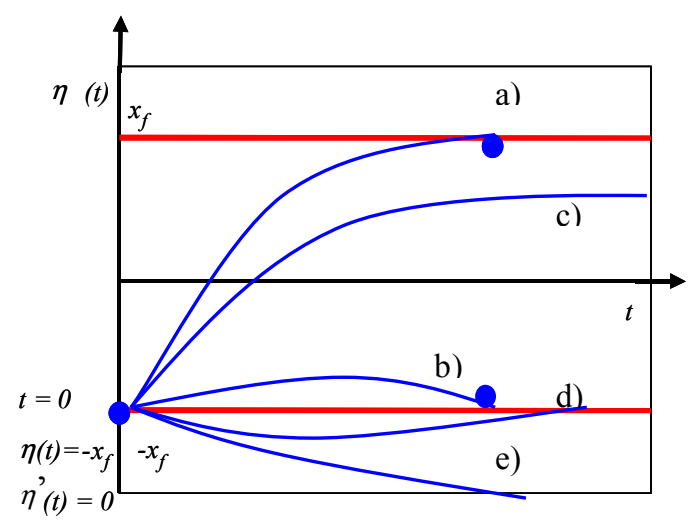

Figura 4. Posibles trayectorias del oscilador sujeto a ruido blanco

En el análisis del modelo sólo las trayectorias definidas en los tres primeros incisos son las que se deben considerar para calcular el valor esperado del cuadrado de la velocidad al primer contacto con las barreras, ya que en el contexto del oscilador no lineal, son los casos que realmente representan una descarga y una nueva fluencia del oscilador.

Con el fin de ilustrar cualitativamente las limitaciones de la solución propuesta por Scharton y Karnopp la figura 5 muestra la función de densidad de probabilidades del tiempo al primer contacto de adentro hacia fuera con alguna de las barreras, para un oscilador con un periodo de vibración $T=0.1$ segundos y dos diferentes niveles de resistencia.

Cabe mencionar que en este estudio la resistencia del oscilador se caracteriza mediante el parámetro $\alpha$, definido como:

$$
\alpha=\frac{R}{m a_{r m s}}
$$


donde $a_{\mathrm{rms}}$ es la aceleración cuadrática media. El valor de $a_{r m s}$ está relacionado con $S_{0}$ conforme a:

$$
a_{r m s}=\sqrt{\frac{2 S_{0}}{\Delta t}}
$$

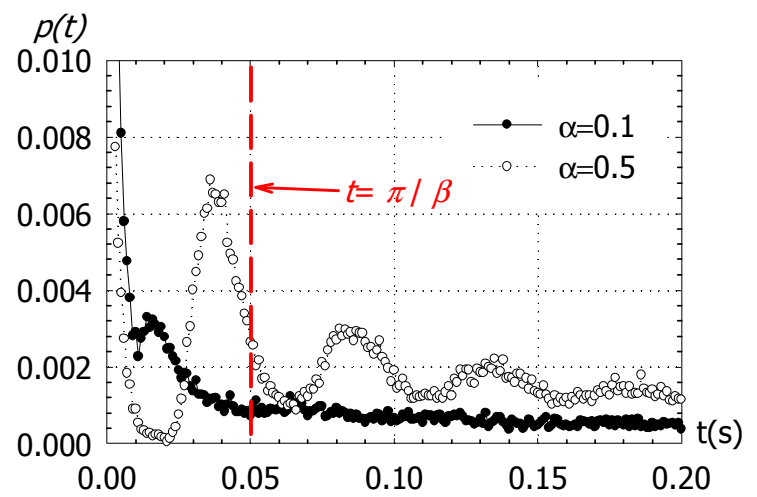

Figura 5. Función de densidad de probabilidad, obtenida con simulaciones, del primer toque del oscilador con alguna de las barreras, $T=0.2 \mathrm{~s}, \xi=0.05$

Las funciones de densidad de probabilidades fueron obtenidas con 100,000 simulaciones. Cabe aclarar que sólo se muestran los primeros 0.2 segundos de las funciones por cuestiones de escala. Con línea gruesa se muestra el valor del tiempo que Karnopp y Scharton consideran como el tiempo en el que ocurre el primer toque con las barreras.

Es claro el efecto que la resistencia tiene en la forma de la función de densidad de probabilidad del primer toque con las barreras. Además, se observa que no es razonable considerar que el tiempo en que se toca por primera vez alguna barrera es independiente de la resistencia.

Conforme a lo discutido en la sección anterior, para estimar la energía histerética disipada por el oscilador se requiere conocer el valor esperado del cuadrado de su velocidad en el instante que toca por primera vez alguna de las barreras y la frecuencia con la que esto ocurre.

Por ello es necesario conocer la función de densidad de probabilidad del primer toque con las barreras. Obtener dicha densidad de probabilidad es un problema clásico en procesos estocásticos que es conocido en inglés como "first passage time problem".

Este es un problema complejo y sólo existen soluciones exactas para procesos de difusión como el proceso de Wiener. En el proceso de Wiener el valor esperado y la varianza del desplazamiento crecen linealmente con el tiempo.

Para el caso del oscilador en estudio las expresiones para los valores esperados y las varianzas del desplazamiento y de la velocidad son bastante más complicadas que las del proceso de Wiener (ver ecuaciones (6), (7), (11) y (12)). 
En este estudio se trató de obtener la expresión exacta para la función de densidad de probabilidad del primer toque con las barreras pero no fue posible debido a complicaciones matemáticas fuertes. En particular, en lo que se refiere a la obtención de la transformada de Laplace de expresiones que involucraban al valor esperado y a la varianza del desplazamiento y de la velocidad.

Sin embargo, se encontró una solución aproximada que permite estimar el valor esperado del cuadrado de la velocidad justo al primer contacto con las barreras y de la frecuencia con lo que esto ocurre, la cual se describe a continuación.

\section{Solución aproximada propuesta}

Se desean obtener las siguientes funciones de densidad de probabilidad:

$p^{+}(t)$ función de densidad de probabilidad del tiempo al primer toque con velocidad positiva con la barrera colocada en $X_{f}$.

$p^{-}(t)$ función de densidad de probabilidad del tiempo al primer toque con velocidad negativa con la barrera colocada en $-X_{f}$.

Como ya se ha discutido anteriormente no fue posible encontrar una expresión exacta que defina estas densidades, aunque es posible calcular la tasa de cruces del desplazamiento por la barrera en $X_{f}$ con velocidad positiva durante el intervalo de tiempo $(t, t+d t)$ conforme a (Roberts, 1968):

$$
v^{+}(t)=\int_{0}^{+\infty}|v| p\left(X_{f}, v\right) d v
$$

donde $v$ es la velocidad en el instante $t \mathrm{y} p\left(X_{f}, v\right)$ es la función de densidad de probabilidad conjunta entre desplazamiento y velocidad, en el instante $t$, evaluada en el desplazamiento igual a $X_{f}$.

La densidad $p\left(X_{f}, v\right)$ puede expresarse como función de la densidad condicional de acuerdo a:

$$
p\left(X_{f}, v\right)=p\left(v \mid \eta=X_{f}\right) p\left(X_{f}\right)
$$

En donde $p\left(v \mid \eta=X_{f}\right)$ es la función de densidad de probabilidad de la velocidad dado que el desplazamiento es igual al desplazamiento de fluencia y $p\left(X_{f}\right)$ es la función de densidad de probabilidad del desplazamiento evaluada en $X_{f}$. Sustituyendo la ecuación (33) en la ecuación (32) se obtiene: 


$$
v^{+}(t)=p\left(X_{f}\right) \int_{0}^{+\infty}|v| p\left(v \mid \eta=X_{f}\right) d v
$$

De esta forma es posible obtener una expresión exacta para $v^{+}(t)$ ya que $p\left(X_{f}\right)$ y $p\left(v \mid \eta=X_{f}\right)$ están definidas por:

$$
\begin{aligned}
& p\left(X_{f}\right)=\frac{1}{\sqrt{2 \pi} \sigma_{\eta t}} e^{-\frac{1}{2} \frac{\left(X_{f}-E[\eta]\right)^{2}}{\sigma_{\eta t}^{2}}} \\
& p\left(v \mid \eta=X_{f}\right)=\frac{1}{\sqrt{2 \pi} S v c^{+}} e^{-\frac{1}{2} \frac{\left(v-E v c^{+}\right)^{2}}{\left(S v c^{+}\right)^{2}}}
\end{aligned}
$$

donde $E v c^{+}$es el valor esperado de la velocidad dado que el desplazamiento es igual a $X_{f}$ y $S v c^{+}$ es la desviación estándar de la velocidad dado que el desplazamiento es igual a $X_{f}$. Estas cantidades están definidas por:

$$
\begin{aligned}
& E v c^{+}=E[\eta(t)]+\left(X_{f}-E[\eta(t)]\right) \frac{\sigma_{\eta t}}{\sigma_{\eta t}} \rho_{t} \\
& S v c^{+}=\sigma_{\eta t} \sqrt{1-\rho_{t}^{2}}
\end{aligned}
$$

Resolviendo la integral de la ecuación (34), con ayuda del programa Maple 8 (2002), y simplificando los términos se obtiene:

$$
v^{+}(t)=\frac{1}{\sqrt{2 \pi} \sigma_{\eta t}} e^{-\frac{1}{2} \frac{\left(X_{f}-E[\eta]\right)^{2}}{\sigma_{\eta t}^{2}}}\left(E v c^{+} \Phi\left(\frac{E v c^{+}}{S v c^{+}}\right)+\frac{S v c^{+}}{\sqrt{2 \pi}} e^{-\frac{1}{2}\left(\frac{E v c^{+}}{S v c^{+}}\right)^{2}}\right)
$$

La tasa de cruces por $-X_{f}$ con velocidad negativa puede obtenerse de manera similar, cambiando los límites de integración en la ecuación (34) de menos infinito a cero y sustituyendo $X_{f}$ por $-X_{f}$.

De esta forma, la expresión para estimar la tasa de cruces del desplazamiento por la barrera en $-X_{f}$ con velocidad negativa durante el intervalo de tiempo $(t, t+d t)$ es:

$$
v^{-}(t)=\frac{1}{\sqrt{2 \pi} \sigma_{\eta t}} e^{-\frac{1}{2} \frac{\left(-X_{f}-E[\eta]\right)^{2}}{\sigma_{\eta t}^{2}}}\left(E v c^{-}\left(\Phi\left(\frac{E v c^{-}}{S v c^{-}}\right)-1\right)+\frac{S v c^{-}}{\sqrt{2 \pi}} e^{-\frac{1}{2}\left(\frac{E v c^{-}}{S v c^{-}}\right)^{2}}\right)
$$


Donde $E v c^{-}$es el valor esperado de la velocidad dado que el desplazamiento es igual a $-X_{f}$ y $S v c^{-}$ es la desviación estándar de la velocidad dado que el desplazamiento es igual a $-X_{f}$, cantidades definidas por:

$$
\begin{aligned}
& E v c^{-}=E[\eta(t)]+\left(-X_{f}-E[\eta(t)]\right) \frac{\sigma_{\eta t}}{\sigma_{\eta t}} \rho_{t} \\
& S v c^{-}=\sigma_{\eta t} \sqrt{1-\rho_{t}^{2}}
\end{aligned}
$$

La figura 6 muestra una comparación entre $p^{+}(t)$ y $v^{+}(t) \cdot p^{+}(t)$ que fue obtenida mediante 50,000 simulaciones y $v^{+}(t)$ con la ecuación (39). La figura 7 compara $p^{-}(t)$ y $v^{-}(t) \cdot p^{-}(t)$ que fue obtenida también mediante 50000 simulaciones y $v^{-}(t)$ con la ecuación $(40)$.

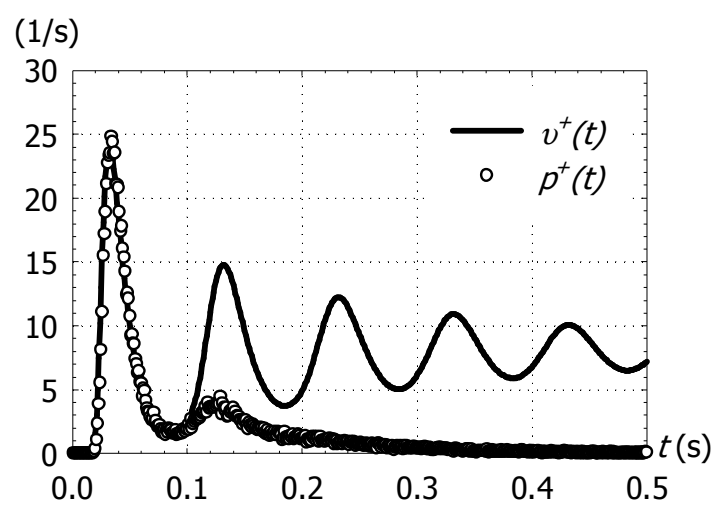

Figura 6. Comparación entre $p^{+}(t)$ y $v^{+}(t) ; T=0.1$ segundos, $\alpha=0.365, \xi=0.05$ y $\Delta t=0.001$ segundos.

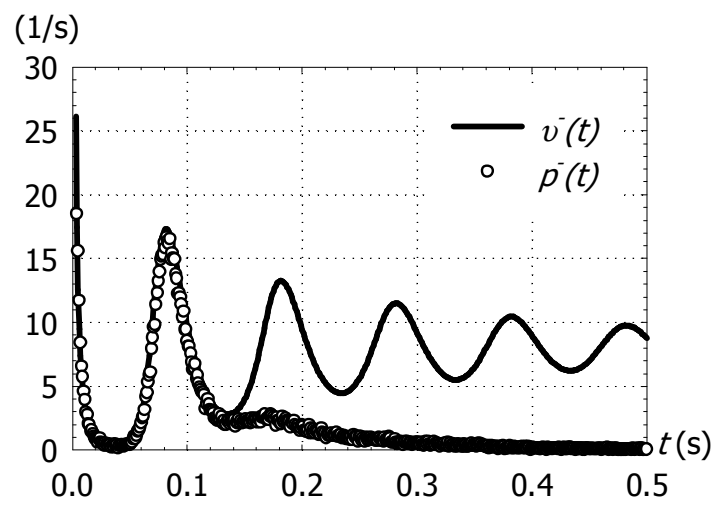

Figura 7. Comparación entre $p^{-}(t)$ y $v^{-}(t) ; T=0.1$ segundos, $\alpha=0.365, \xi=0.05$ y $\Delta t=0.001$ segundos. 
Como puede observarse, para valores de $t$ cercanos a cero $p^{+}(t)$ y $v^{+}(t)$; y $p^{-}(t)$ y $v^{-}(t)$ son prácticamente iguales y conforme el tiempo se incrementa adquieren valores muy diferentes. Cuando el tiempo tiende a infinito $p^{+}(t)$ y $p^{-}(t)$ tienden a cero mientras que $v^{+}(t)$ y $v^{-}(t)$ tienden a un valor constante.

La interpretación física de esta observación es la siguiente: las ecuaciones (39) y (40) proporcionan una estimación del número de veces que se cruzan las barreras en un intervalo de tiempo $d t$, cuando el valor de $t$ es cercano a cero; es probable que estos cruces correspondan a la primera vez que se toca la barrera. Conforme el tiempo crece probablemente ya se habrá cruzado anteriormente alguna de las barreras.

En las figuras 6 y 7 se observa también que las modas de $p^{+}(t)$ y $p^{-}(t)$ coinciden con los primeros máximos de las funciones $v^{+}(t)$ y $v^{-}(t)$ respectivamente.

Para valores de $t$ cercanos a cero los valores obtenidos con la ecuación (40) crecen rápidamente, como puede observarse en la figura 7. Cuando $t$ es exactamente igual a cero la ecuación (40) se indetermina y en el límite tiende a infinito. Esta particularidad se debe a que el coeficiente de correlación entre velocidad y desplazamiento tiene una discontinuidad en $t$ igual a cero, como puede observarse en la ecuación (13).

Las representaciones de $p^{+}(t)$ y $p^{-}(t)$ mostradas en las figuras 6 y 7 proporcionan la siguiente información, que al ser complementada con ciertas hipótesis permite obtener la solución propuesta:

- Para $t$ cercano a cero no se presentan contactos con la barrera positiva; sólo existen trayectorias del tipo $b$ ).

- Una vez que comienzan a presentarse contactos con la barrera positiva estos tienden a ocurrir con mayor frecuencia que los contactos con la barrera negativa.

- Las trayectorias del tipo b) tienden a concentrarse en dos grupos: el grupo más numeroso tiende a presentarse cuando $t$ es muy pequeño y el segundo grupo se presenta después de que ocurrió la moda de las trayectorias tipo a).

Con base en estas observaciones se puede estimar la probabilidad de que ocurran trayectorias a) y trayectorias b) de acuerdo con lo siguiente:

Sea $t_{l}$ el tiempo que transcurre para que se presente el primer contacto con la barrera positiva, es decir el tiempo que debe pasar para que $p^{+}(t)$ sea mayor a cero. Si se considera que todas las trayectorias del tipo b) ocurren en los primeros $t_{l}$ segundos, la probabilidad de ocurrencia de trayectorias b) $(P(B))$ puede calcularse como:

$$
P(B)=\int_{0}^{t_{1}} p^{-}(t) d t
$$


Suponiendo que la probabilidad de que el oscilador no toque ninguna barrera es nula (esta suposición es verdadera para el caso de ruido blanco teórico, ya que como este tiene una duración infinita siempre existe la posibilidad de que se toque alguna barrera) la probabilidad de ocurrencia de trayectorias a) $(P(A))$ queda definida por:

$$
P(A)=1-P(B)
$$

El problema para aplicar las ecuaciones (43) y (44) es que no se cuenta con una expresión para $p^{-}(t)$, pero suponiendo que $p^{-}(t)$ puede aproximarse razonablemente con la función $v^{-}(t)$ siempre que $t_{1}$ sea pequeño, entonces $P(B)$ puede aproximarse con:

$$
P(B) \approx \int_{t_{0}}^{t_{1}} v^{-}(t) d t
$$

Note que en la ecuación (45) se ha cambiado el límite inferior de integración, respecto a la ecuación (43), debido a que la función $v^{-}(t)$ tiene una discontinuidad en $t$ igual a cero; en este estudio se observó que el efecto de la discontinuidad tiende a suavizarse para un valor de $t_{0}$ igual a 0.002 segundos.

Con las ecuaciones (45) y (44) es posible estimar la proporción con la que ocurren los contactos con las barreras positiva y negativa respectivamente.

Ahora, la función $p^{+}(t)$ se idealiza como una delta de Dirac localizada en un tiempo igual a $t_{f}$ que se define como el tiempo en el que se presenta el primer máximo en la función $v^{+}(t)$.

$$
p^{+}(t) \approx \delta\left(t-t_{f}\right)
$$

Aunque se ha discutido que la función $p^{-}(t)$ puede aproximarse por la función $v^{-}(t)$ para valores pequeños de $t$, en este punto se idealiza como una delta de Dirac localizada en un tiempo igual a cero para simplificar la obtención del valor esperado del cuadrado de la velocidad al primer contacto con la barreras negativa. Cabe aclarar que esta suposición no se utiliza en el cálculo del número de contactos con la barrera negativa.

$$
p^{-}(t) \approx \delta(t)
$$

Por otro lado, el valor esperado del cuadrado de la velocidad dado que se cruza la barrera en $X_{f}$ con velocidad positiva $\left(\mathrm{E}\left[v^{2}\right]^{+}\right)$es igual a (los detalles de la obtención de esta ecuación se muestran en el anexo A):

$$
E\left[v^{2}\right]^{+}=\left(E v c^{+}\right)^{2}+\left(S v c^{+}\right)^{2}+\frac{E v c^{+} S v c^{+}}{\sqrt{2 \pi} \Phi\left(\frac{E v c^{+}}{S v c^{+}}\right)} e^{-\frac{1}{2}\left(\frac{E v c^{+}}{S v c^{+}}\right)^{2}}
$$


De esta forma, es posible estimar el valor esperado del cuadrado de la velocidad dado que se toca la barrera en $X_{f}$ con velocidad positiva conforme a:

$$
\begin{aligned}
& E\left[v_{f}^{2}\right]^{+}=\int_{0}^{\infty} E\left[v^{2}\right]^{+} \delta\left(t-t_{f}\right) d t \\
& E\left[v_{f}^{2}\right]^{+}=E\left[v^{2}\left(t=t_{f}\right)\right]^{+}
\end{aligned}
$$

Es decir, el valor de $\mathrm{E}\left[v_{f}^{2}\right]^{+}$se obtiene evaluando al ecuación (48) para $t$ igual a $t_{f}$.

Con el mismo razonamiento se puede obtener el valor esperado del cuadrado de la velocidad dado que se toca la barrera en $-X_{f}$ con velocidad negativa. Pero, como la delta de Dirac se coloca en cero (Ecuación (47)) el valor esperado del cuadrado de la velocidad también es igual a cero.

$$
E\left[v_{f}^{2}\right]^{-}=\int_{-\infty}^{0} E\left[v^{2}\right]^{-} \delta(t) d t=E\left[v^{2}(t=0)\right]^{-}=0
$$

Finalmente, el valor esperado del cuadrado de la velocidad dado que se toca alguna barrera de adentro hacia fuera queda definido por:

$$
E\left[v_{f}^{2}\right]=\frac{E\left[v_{f}^{2}\right]^{+} P(A)+E\left[v_{f}^{2}\right]^{-} P(B)}{P(A)+P(B)}=E\left[v_{f}^{2}\right]^{+} P(A)
$$

Suponiendo que la energía cinética que tiene el oscilador al inicio de cada fluencia se disipa completamente en energía histerética durante el tiempo que tarda en descargar, es posible estimar el valor esperado de la energía histerética por unidad de masa disipada en cada fluencia $\left(\mathrm{E}\left[\Delta E_{H \mu}\right]\right)$ conforme a:

$$
E\left[\Delta E_{H \mu}\right]=\frac{1}{2} E\left[v_{f}^{2}\right]
$$

Conocido el valor de $\mathrm{E}\left[\Delta E_{H \mu}\right]$ es posible estimar el valor esperado de la energía histerética por unidad de masa $\left(E_{H \mu}\right)$ disipada por el oscilador para cierta duración $\left(t_{d}\right)$ del ruido blanco conforme a:

$$
E\left\lfloor E_{H \mu}\right\rfloor=E\left\lfloor\Delta E_{H \mu}\right\rfloor E\left\lfloor n_{f}\right\rfloor
$$

donde $\mathrm{E}\left[n_{f}\right]$ es el valor esperado del número de fluencias en una duración del ruido blanco igual a $t_{d}$. 
De acuerdo con lo discutido durante esta sección, el valor de $\mathrm{E}\left[n_{f}\right]$ se puede obtener con:

$E\left[n_{f}\right]=E\left[n_{f}\right]^{+}+E\left[n_{f}\right]^{-}$

donde $\mathrm{E}\left[n_{f}\right]^{+}$y $\mathrm{E}\left[n_{f}\right]^{-}$son el valor esperado del número de fluencias con trayectorias a) y b) respectivamente.

El valor de $\mathrm{E}\left[n_{f}\right]^{+}$se obtiene con la siguiente expresión:

$$
E\left[n_{f}\right]^{+}=\int_{0}^{t_{d}} v^{+}(t) d t
$$

El valor de $\mathrm{E}\left[n_{f}\right]$ - se obtiene de forma diferente debido a la discontinuidad que se presenta en la función $v^{-}(t)$ cuando $t$ es igual a cero.

$$
E\left[n_{f}\right]^{-} \approx \int_{t_{1}}^{t_{d}} v^{-}(t) d t+\frac{P(B)}{P(A)} E\left[n_{f}\right]^{+}
$$

Se mencionó anteriormente que las trayectorias del tipo b) tienden a concentrarse en dos grupos: el grupo más numeroso tiende a presentarse cuando $t$ es muy pequeño y el segundo grupo se presenta después de que ocurrió la moda de las trayectorias tipo a). Dentro de este contexto, en la ecuación (57) el segundo término representa los casos b) del primer grupo y el primer término representa los casos restantes.

Se decidió adoptar esta forma de estimar el número de casos b) para evitar complicaciones numéricas en la obtención de la integral de $v^{-}(t)$ debido a la discontinuidad que dicha función tiene para $t$ cercano a cero.

Cabe mencionar que la ecuación (57) supone que la probabilidad de que ocurra un contacto con la barrera es igual a la unidad, lo cual pudiera no ser válido para duraciones muy cortas respecto al periodo del sistema y altos niveles de resistencia.

A continuación se presenta un resumen de los pasos del método propuesto:

1) Son conocidos: el periodo de la estructura $(T)$, la resistencia del sistema por unidad de masa $(R / m)$, el coeficiente de amortiguamiento viscoso $(\xi)$, la densidad espectral de potencia del ruido $\left(S_{0}\right)$, la duración del ruido $\left(t_{d}\right)$ y el ancho de banda del ruido caracterizado mediante el $\Delta t$ con que se genera el ruido blanco discreto.

2) Calcular el valor del desplazamiento inicial de fluencia del sistema $\left(X_{f}\right)$.

3) Estimar el valor de $t_{l}$, que es el valor mínimo positivo de $t$ que hace que la ecuación (39) sea distinta de cero.

4) Calcular el valor de $P(B)$ mediante la ecuación (45). Si $t_{1}$ es menor que $t_{0} P(B)$ se considera igual a cero.

5) Estimar el valor de $P(A)$ con la ecuación (44). 
6) Encontrar el valor de $t_{f}$, que es valor de $t$ en el que se presenta el valor máximo de la ecuación (39).

7) Estimar el valor de $\mathrm{E}\left[v_{f}^{2}\right]^{+}$sustituyendo el valor de $t_{f}$ en la ecuación (50).

8) Calcular el valor de $\mathrm{E}\left[v_{f}^{2}\right]$ con la ecuación (52).

9) Obtener el valor de $\mathrm{E}\left[\Delta E_{H \mu}\right]$ mediante la ecuación (53).

10) Calcular el valor de $\mathrm{E}\left[n_{f}\right]^{+}$con la ecuación (56).

11) Calcular el valor de $\mathrm{E}\left[n_{f}\right]^{-}$con la ecuación (57).

12) Calcular el valor de $\mathrm{E}\left[n_{f}\right]$ con la ecuación (55).

13) Calcular el valor de $\mathrm{E}\left[\mathrm{E}_{H \mu}\right]$ mediante la ecuación (54).

Debido a la complejidad algebraica de las ecuaciones (39) y (40) los pasos 3, 4, 6, 10 y 11 tienen que resolverse numéricamente.

\section{EVALUACIÓN DEL MÉTODO PROPUESTO}

Para evaluar el desempeño de las expresiones propuestas se calculó la respuesta dinámica de osciladores elástoplasticos sujetos a 1000 acelerogramas de ruido blanco gaussiano discreto, con duración de 16.384 segundos y $\Delta t$ de 0.001 . Se consideraron periodos de 0.1 a 6 segundos y resistencias, caracterizadas a través del parámetro $\alpha$, de 0.01 a 3 .

Los resultados obtenidos con las simulaciones se compararon con los estimados mediante el procedimiento descrito en la sección anterior y con las expresiones propuestas por Karnopp y Scharton (1966). Los resultados se muestran en las figuras 8 a 11. En dichas figuras, con línea continua se muestran los resultados obtenidos con el procedimiento propuesto en este estudio (E), con línea discontinua los resultados obtenidos con las expresiones de Karnopp y Scharton (KS) y con círculos los valores promedio observados en las simulaciones.

El método de Karnopp y Scharton se aplicó con el siguiente criterio: en sistemas con valores del parámetro $a$ menores a la unidad se utilizó el procedimiento definido por las ecuaciones 23 a 27, mientras que los casos con valores de $a$ mayores a la unidad se aplicó la solución estacionaria (ecuaciones 28 y 29 de Karnopp y Scharton, 1966).

La figura 8 muestra una comparación entre valores de $\mathrm{E}\left[v_{f}^{2}\right]$ calculados mediante los métodos considerados y los obtenidos con las simulaciones. Como puede observarse, las expresiones propuestas conducen a estimaciones razonables de $\mathrm{E}\left[v_{f}^{2}\right]$, excepto para el caso de resistencias muy bajas $(\alpha=0.01)$ donde el método propuesto tiende a sobrestimar los valores observados en las simulaciones.

En general, se observan mejoras en las estimaciones obtenidas con el método propuesto respecto al método de Karnopp y Scharton, excepto para el caso de $\alpha=0.01$. Puede observarse, que el método de Karnopp y Scharton conduce a estimaciones razonables de $\mathrm{E}\left[v_{f}^{2}\right]$, excepto para sistemas con $\alpha=0.1$, donde subestima el valor de $\mathrm{E}\left[v_{f}^{2}\right]$. 
Note que para el método de Karnopp y Scharton aparece una discontinuidad para un valor de $T$ de 1.2 segundos y $\alpha=0.25$. Esta discontinuidad se debe a que para periodos mayores a 1.2 segundos, con $\alpha=0.25$, se presentan valores de $a$ mayores a la unidad y por lo tanto se aplica la solución estacionaria, mientras que para periodos menores se tienen valores de $a$ menores a la unidad y se aplican las ecuaciones 23 a 27. Esta discontinuidad también aparece en las figuras 9 a 11 .
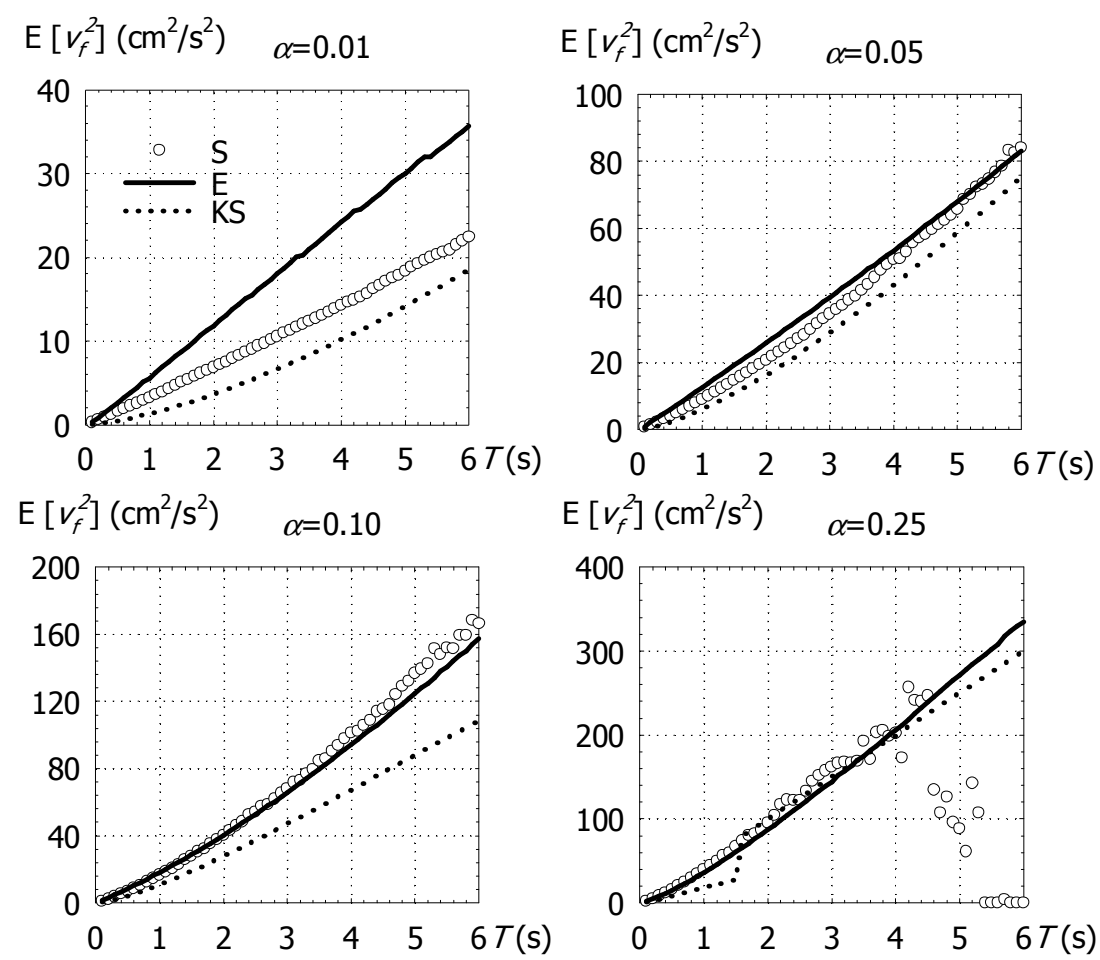

$\mathrm{E}\left[v_{f}^{2}\right]\left(\mathrm{cm}^{2} / \mathrm{s}^{2}\right) \quad \alpha=0.25$
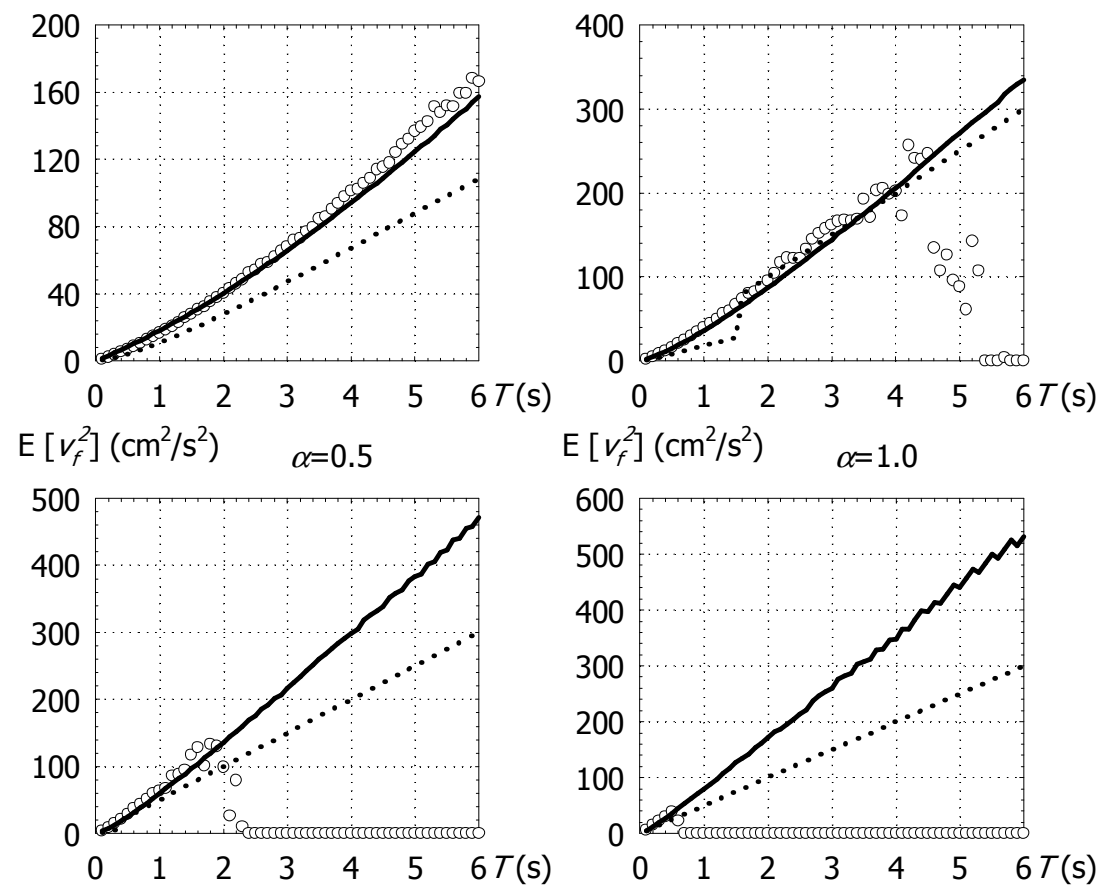

Figura 8. Comparación entre diferentes estimaciones de $\mathrm{E}\left[v_{f}^{2}\right]$ y el valor calculado con simulaciones, $\xi=0.05$.

Como puede observarse, a partir de $\alpha=0.25$ se presentan dispersiones importantes en los valores de $\mathrm{E}\left[v_{f}^{2}\right]$ observados en las simulaciones para periodos largos; esto se debe a que para estas combinaciones de periodo y resistencia es muy poco probable que se presenten fluencias 
para la duración considerada. Es decir, las diferencias en resistencias altas y periodos largos se deben a la falta de simulaciones.
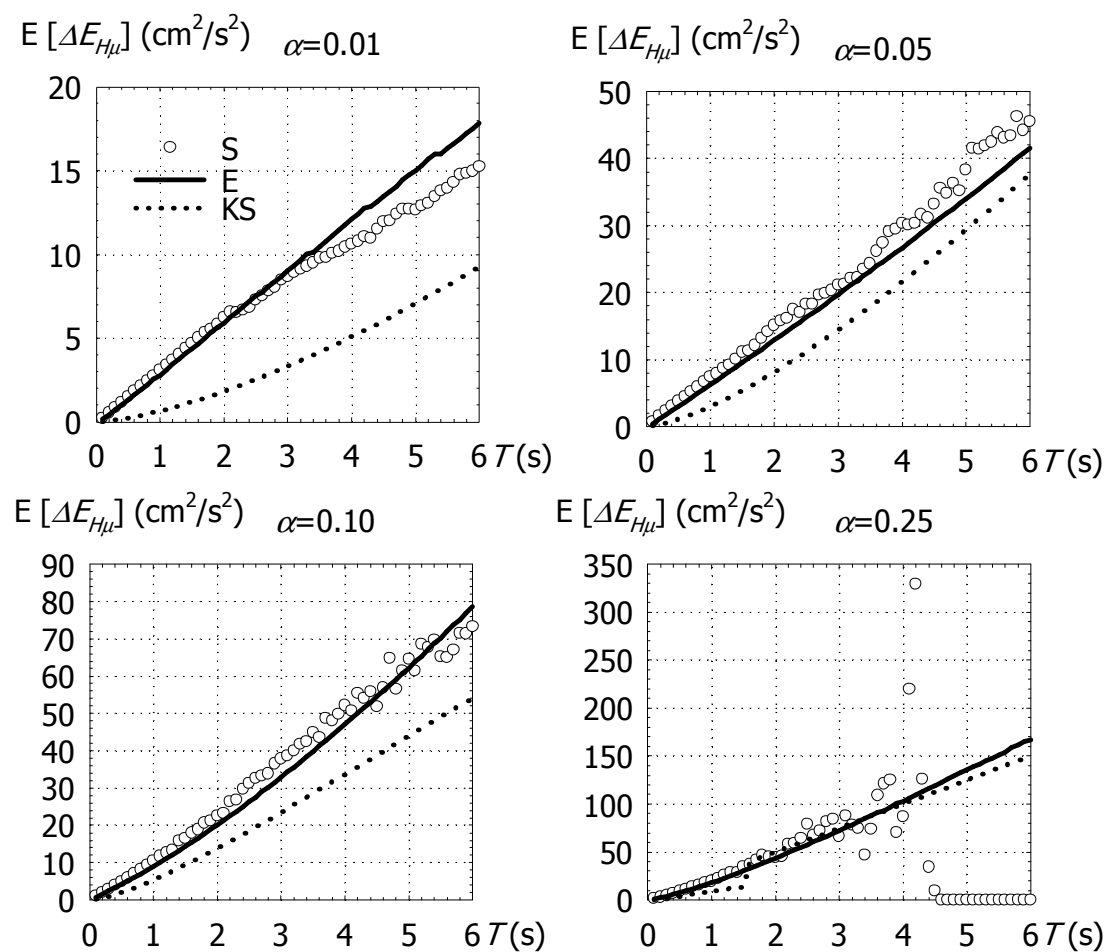

$\mathrm{E}\left[\Delta E_{H \mu}\right]\left(\mathrm{cm}^{2} / \mathrm{s}^{2}\right) \quad \alpha=0.25$
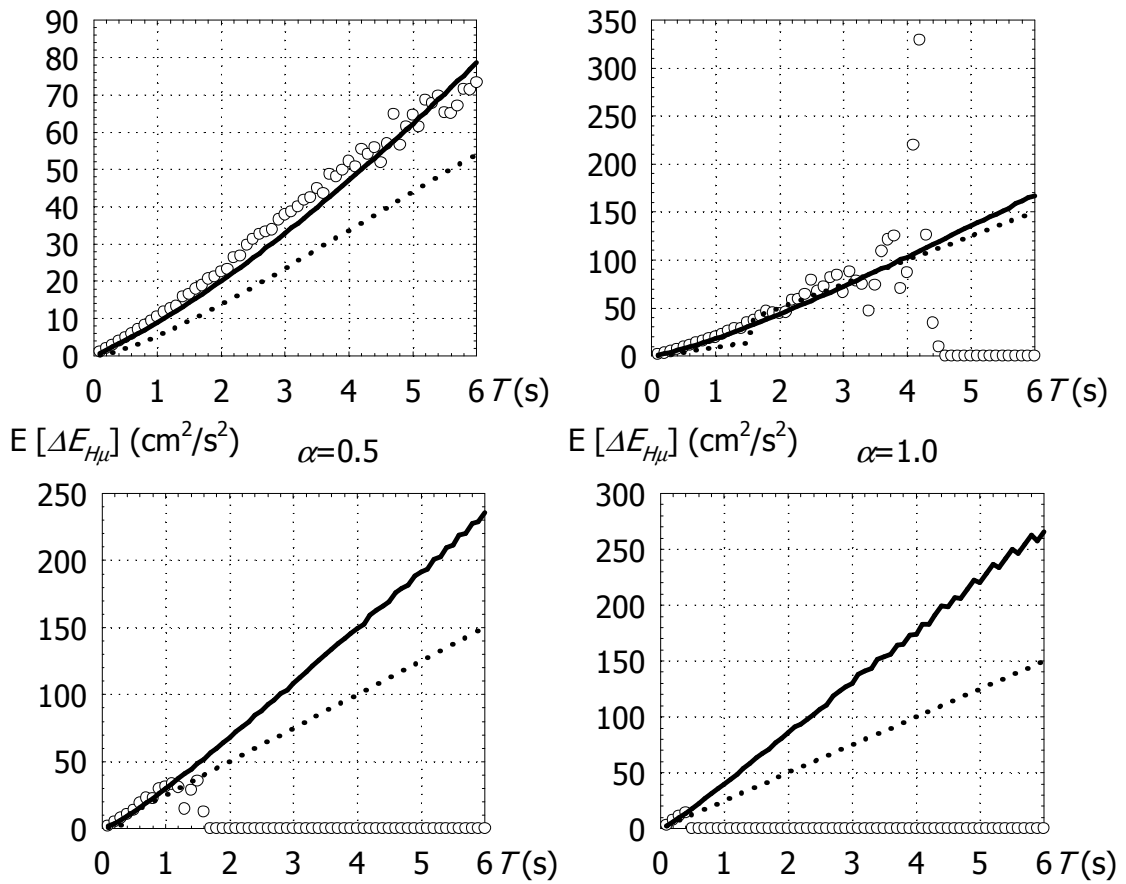

Figura 9. Comparación entre diferentes estimaciones de $\mathrm{E}\left[\Delta E_{H \mu}\right]$ y el valor calculado con simulaciones, $\xi=0.05$.

En cambio, las diferencias en resistencias muy bajas se deben a que en cálculo de $P(B)$ mediante la ecuación (45) sólo se consideran los contactos con las barrera en - $X_{f}$ que ocurren entre $t_{0}$ y $t_{1}$ segundos.

Si la resistencia es muy baja el valor de $t_{l}$ tenderá a ser muy pequeño y es muy probable que después de $t_{l}$ segundos todavía se sigan presentando contactos con la barrera con $-X_{f}$, los cuáles tendrían una velocidad muy pequeña y no son considerados. Cabe mencionar, que sistemas con $\alpha=0.01$ no están dentro del campo de interés de la ingeniería sísmica pues se observó, en las simulaciones, que tendrían demandas de ductilidad máxima del orden de $10^{4}$. 
La figura 9 compara los valores de $\mathrm{E}\left[\Delta E_{H \mu}\right]$ calculados mediante los métodos considerados en esta sección y los obtenidos con las simulaciones. Como puede observarse, las expresiones propuestas conducen a estimaciones razonables de $\mathrm{E}\left[\Delta E_{H \mu}\right]$; las mayores diferencias se observan para sistemas con $\alpha=0.01$ y periodos mayores a 3 segundos. En estos sistemas se sobrestima ligeramente el valor de $\mathrm{E}\left[\Delta E_{H \mu}\right]$ debido a la sobrestimación en $\mathrm{E}\left[v_{f}^{2}\right]$, mientras que para resistencias altas y periodos largos se observa también la falta de simulaciones.
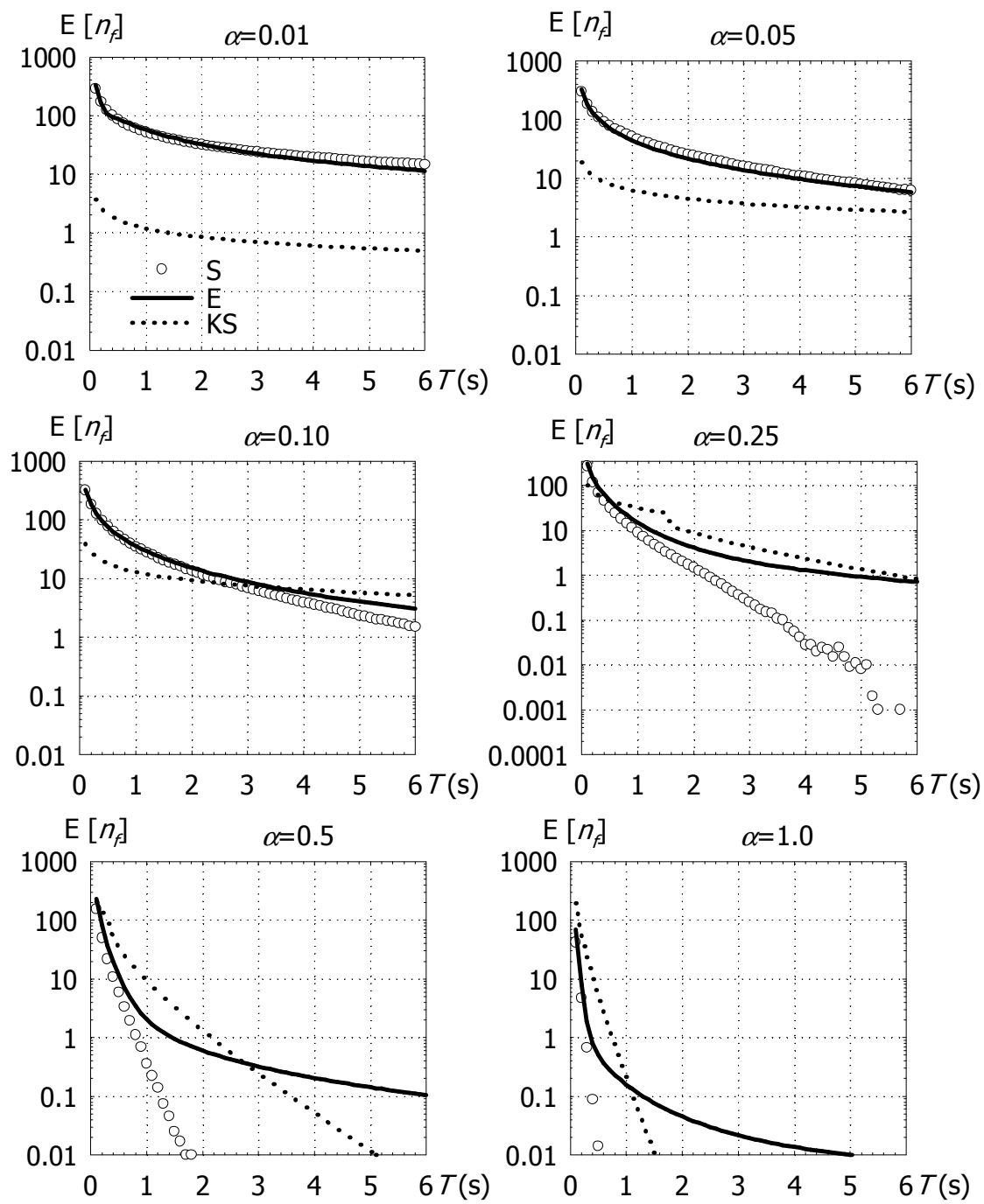

Figura 10. Comparación entre diferentes estimaciones de $\mathrm{E}\left[n_{f}\right]$ y el valor calculado con simulaciones, $\xi=0.05$ y $t_{d}=16.384$ segundos.

Como puede observarse, el método de Karnopp y Scharton conduce a altos niveles de error para sistemas con baja resistencia $(\alpha=0.01)$, ya que subestima considerablemente el valor de $\mathrm{E}\left[\Delta E_{H \mu}\right]$. El nivel de error disminuye al aumentar el valor de $\alpha$ y a partir de $\alpha=0.25$ se tiene el mismo nivel de aproximación que el método propuesto. 
La figura 10 compara los valores observados en las simulaciones de $\mathrm{E}\left[n_{f}\right]$ con aquellos obtenidos con el método presentado. Se observa una excelente coincidencia para valores de E $\left[n_{f}\right]$ mayores a la unidad, mientras que para valores menores a la unidad el método propuesto tiende a sobrestimar dichos valores. Esta sobrestimación se debe a que las expresiones propuestas suponen que la probabilidad de que el oscilador fluya es igual a la unidad.

Cabe mencionar que en el contexto de la ingeniería sísmica los casos de interés son aquellos en los que, por lo menos, $\mathrm{E}\left[n_{f}\right]$ es igual a la unidad, ya que valores menores indican que el sistema en promedio tiende a permanecer elástico y, por lo tanto, sin daño.

Es en este parámetro donde se observa claramente la diferencia entre el método presentado y las expresiones propuestas por Karnopp y Scharton. Para sistemas con baja resistencia $(\alpha=0.01)$ el método de Karnopp y Scharton subestima considerablemente el número de fluencias que experimenta el oscilador. Esta subestimación del número de fluencias se debe a que la propuesta de Karnopp y Scharton considera que el contacto con las barreras tiende a ocurrir a cada $\pi / \omega_{d}$ segundos. Para resistencias bajas el primer contacto con las barreras tiende a ocurrir en tiempos bastante menores que $\pi / \omega_{d}$, tal como puede observarse en la figura 5. Note como el nivel de error del método de Karnopp y Scharton tiende a disminuir al aumentar el valor de $\alpha$.

Cabe aclarar que el método de Karnopp y Scharton fue desarrollado para sistemas con muy altas frecuencias de vibración (del orden de $1600 \mathrm{rad} / \mathrm{s}$ ) y valores altos de $\alpha$, por lo que al aplicarlo a sistemas para los que no fue desarrollado se presentan las grandes diferencias que se muestran en las figuras 10 y 11. El objetivo de las comparaciones de esta sección es mostrar que, para sistemas con propiedades comunes en la ingeniería estructural, es necesario contar con una buena estimación del valor de la densidad de probabilidad del tiempo al primer contacto con las barreras para obtener una aproximación razonable en las demandas de energía histerética.

Finalmente, la figura 11 compara los espectros de $\mathrm{E}\left[E_{H \mu}\right]$ obtenidos mediante simulaciones y los obtenidos con los métodos considerados. En general se observa una buena aproximación del procedimiento propuesto. Para $\alpha=0.01,0.05$ y 0.1 las diferencias se atribuyen a que la energía cinética del oscilador en la fluencia no se disipa completamente en energía plástica durante la fluencia como se supone en la ecuación (53) y a las otras suposiciones utilizadas.

Con el método de Karnopp y Sharton se obtienen estimaciones razonables sólo para periodos muy cortos y altos niveles de resistencia. Para valores pequeños de $\alpha$ se obtienen subestimaciones importantes. La causa principal de los errores del método de Karnopp y Scharton, mostrados en la figura 11, se deben a los altos niveles de error que se obtienen en el número de fluencias del oscilador. 

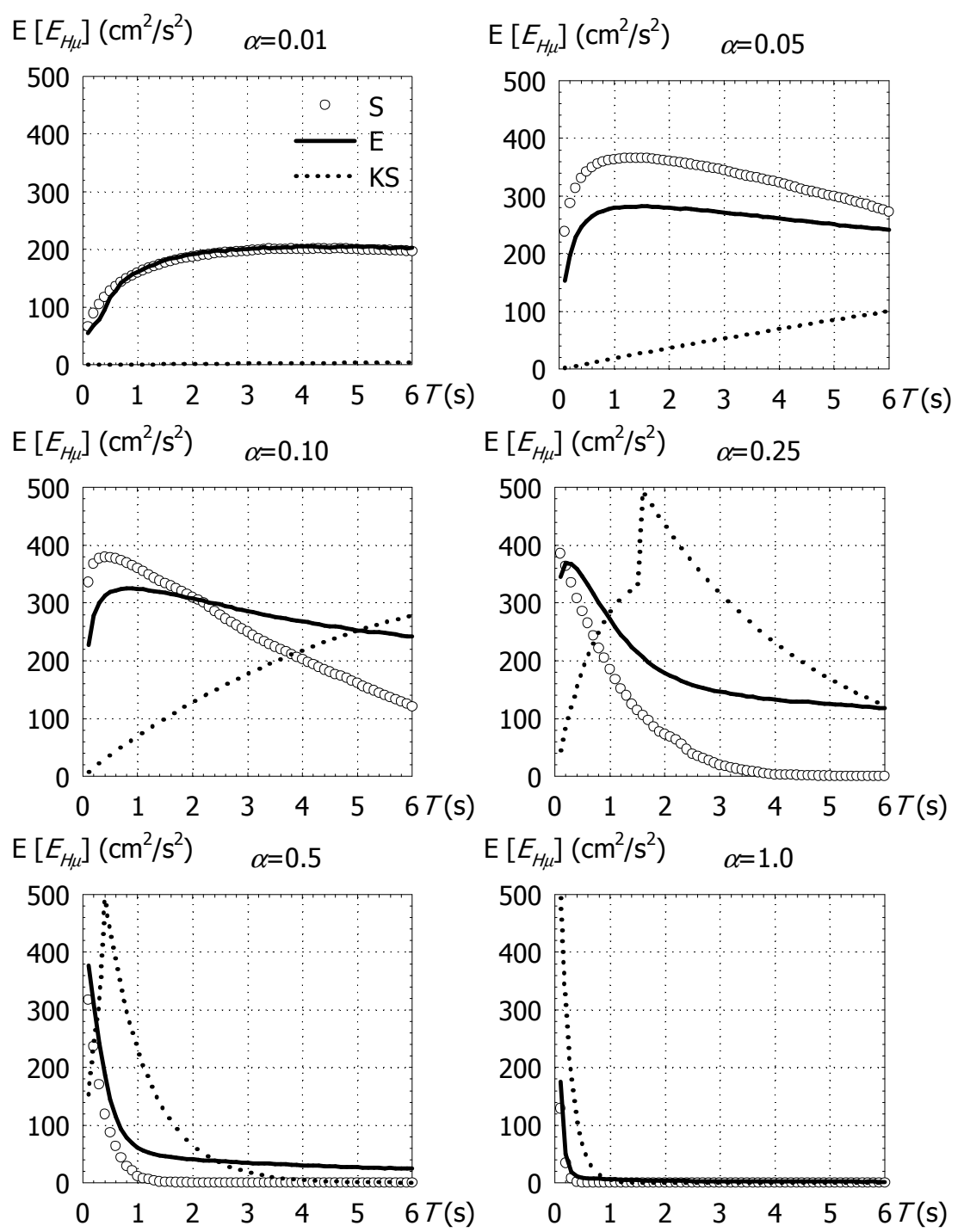

Figura 11. Comparación entre diferentes estimaciones de $\mathrm{E}\left[E_{H \mu}\right]$ y el valor calculado con simulaciones, $\xi=0.05$ y $t_{d}=16.384$ segundos.

\section{APLICACIONES EN OSCILADORES ELASTOPLÁSTICOS SUJETOS A EXCITACIONES SÍSMICAS DE BANDA ANCHA}

El método obtenido en la sección anterior fue desarrollado para ruido blanco gaussiano. Sin embargo, bajo ciertas condiciones, es aplicable a excitaciones sísmicas de banda ancha.

Para aplicar el método propuesto es necesario caracterizar a la aceleración del suelo mediante un ruido blanco gaussiano equivalente. La información necesaria para definir el ruido blanco equivalente se encuentra en el espectro de amplitudes de Fourier de la aceleración del movimiento sísmico. 
El procedimiento para obtener las propiedades del ruido blanco equivalente se describe a continuación, partiendo de que el espectro de amplitudes de Fourier de la aceleración es conocido.

1) Determinar la banda de frecuencias en las que la solución de ruido blanco es aplicable. Dicha banda de frecuencias queda definida por la frecuencia mínima $\left(f_{\min }\right)$ y la frecuencia máxima $\left(f_{\max }\right)$ tal como se muestra en la figura 12. Los valores de $f_{\min } \mathrm{y} f_{\max }$ se definen como los límites en los que el espectro de amplitudes de Fourier tiende a permanecer constante. Cabe mencionar que para definir el ancho de banda se trabajo sólo con la parte del espectro entre 0.1 y $10 \mathrm{~Hz}$ que es la banda de frecuencias en la que normalmente se encuentran las estructuras.

2) El valor de $f_{\max }$ obtenido en el inciso 1) define el $\Delta t$ del ruido blanco equivalente conforme a:

$$
\Delta t=\frac{1}{2 f_{\max }}
$$

3) Calcular el valor esperado del espectro de amplitudes de Fourier $\mathrm{E}[|A(\omega)|] \mathrm{y}$ del cuadrado del espectro de amplitudes de Fourier $\mathrm{E}\left[|A(\omega)|^{2}\right]$, en la banda considerada, conforme a:

$$
\begin{aligned}
& E[A(\omega)]=\frac{1}{n_{f}} \sum_{f_{\min }}^{f_{\max }}|A(\omega)|_{i} \\
& E\left[\left.A(\omega)\right|^{2}\right]=\frac{1}{n_{f}} \sum_{f_{\min }}^{f_{\max }}|A(\omega)|_{i}^{2}
\end{aligned}
$$

donde $n_{f}$ es el número de frecuencias contenido en la banda definida por $f_{\min } \mathrm{y} f_{\max } \mathrm{y}$ $|A(\omega)|_{i}$ es el valor del espectro de amplitudes de Fourier para la i-ésima frecuencia contenida en dicha banda.

4) Generar una señal de ruido blanco suponiendo alguna duración $\left(t_{d}\right)$ conforme a:

$$
a(t)=\sqrt{-2 \ln \left(u_{1}\right)} \sin \left(2 \pi u_{2}\right) \sqrt{\frac{E\left[A(\omega)^{2}\right]}{\Delta t t_{d}}}
$$

donde $u_{1}$ y $u_{2}$ son dos números aleatorios con distribución uniforme entre cero y uno.

5) Calcular el espectro de Fourier de la señal generada; si la transformada de Fourier de dicha señal es igual al valor de $\mathrm{E}[|A(\omega)|]$ obtenido en el inciso 3) la duración del ruido blanco equivalente es la que se utilizó en la ecuación 61. En caso de que el espectro de 
Fourier de la señal generada no coincida con el $\mathrm{E}[|A(\omega)|]$ obtenido en el inciso 3) se debe proponer un nuevo valor de duración y generar una nueva señal hasta que ambos valores coincidan.

Aunque se trata de un proceso iterativo, el cálculo de la duración del ruido equivalente converge rápidamente si se toma como valor inicial la duración del acelerograma calculada conforme a Trifunac y Brady (1975). Para la muestra de acelerogramas utilizada mas adelante se observó que la duración del ruido equivalente era prácticamente igual a la duración calculada conforme a la definición de Trifunac y Brady (1975).

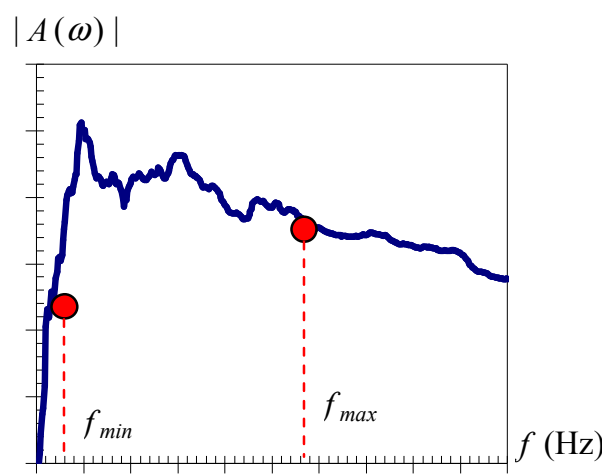

Figura 12. Definición del ancho de banda de un espectro de amplitudes de Fourier de un movimiento sísmico

Una vez conocidos los valores de $\mathrm{E}\left[|A(\omega)|^{2}\right]$ y de la duración del ruido blanco equivalente el valor de $S_{0}$ a utilizar en el método propuesto se estima de acuerdo a:

$$
S_{0}=\frac{E\left\lfloor\left. A(\omega)\right|^{2}\right]}{2 t_{d}}
$$

Cabe mencionar que el método propuesto sólo es válido en el intervalo de frecuencias delimitado por $f_{\max } \mathrm{y} f_{\min } \mathrm{y}$ fuera de dicho intervalo no es aplicable.

Para el caso de acelerogramas reales, si la frecuencia del sistema tiende a infinito la energía histerética tiende a cero independientemente de la resistencia del sistema. Con base en este límite teórico se propone la siguiente expresión para calcular la energía histerética disipada por osciladores elastoplásticos sujetos a acelerogramas de banda ancha:

$$
E\left[E_{H \mu}\right]=\left\{\begin{array}{l}
T f_{\max } E\left[E_{H \mu}\right]_{f \max } \quad \text { Si } T<1 / f_{\max } \\
E\left[\Delta E_{H \mu}\right] E\left[n_{f}\right] \quad \text { Si } 1 / f_{\max } \leq T \leq 1 / f_{\min }
\end{array}\right.
$$

donde $E\left[\Delta E_{H \mu}\right]$ y $E\left[n_{f}\right]$ se calculan con el método descrito en la sección anterior y $E\left[\Delta E_{H \mu}\right]$ fmax es el valor que se obtiene al evaluar el método propuesto para un valor de $T$ igual $1 / f_{\max }$. 
Cabe aclarar que no existe una razón teórica para que a partir de $f_{\max }$ el valor de $\mathrm{E}\left[E_{H \mu}\right]$ disminuya linealmente hasta cero; simplemente se decidió utilizar la variación mas sencilla posible.

El procedimiento descrito se aplicó para estimar la energía histerética disipada por osciladores sujetos a una muestra de acelerogramas de banda ancha. La tabla 1 lista los diferentes acelerogramas considerados. La tabla incluye los valores de la aceleración máxima del suelo $\left(A_{\max }\right)$, así como la duración calculada conforme a Trifunac y Brady (1975) que para la muestra considerada coincide con la duración del ruido blando equivalente.

Tabla 1. Acelerogramas de banda ancha considerados

\begin{tabular}{|l|l|l|l|l|l|l|l|}
\hline Fecha & Estación & Comp & Ms & $\begin{array}{l}f_{\min } \\
(\mathrm{Hz})\end{array}$ & $\begin{array}{l}f_{\max } \\
(\mathrm{Hz})\end{array}$ & $\begin{array}{l}t_{d} \\
(\mathrm{~s})\end{array}$ & $\begin{array}{l}A_{\max } \\
\left(\mathrm{cm} / \mathrm{s}^{2}\right)\end{array}$ \\
\hline $09 / 11 / 74$ & PRQ & EW & 7.2 & 0.2 & 5.96 & 18 & 46.2 \\
\hline $09 / 11 / 74$ & PRQ & NS & 7.2 & 0.2 & 4.68 & 14 & 69.2 \\
\hline $20 / 09 / 99$ & HWA023 & NS & 7.6 & 0.1 & 2.8 & 17 & 36.4 \\
\hline $20 / 09 / 99$ & HWA023 & EW & 7.6 & 0.1 & 2.7 & 23 & 36.7 \\
\hline $18 / 10 / 89$ & Hollister SV & 270 & 7.1 & 0.1 & 2.8 & 16 & 35.4 \\
\hline $18 / 10 / 89$ & Hollister SV & 360 & 7.1 & 0.1 & 2.7 & 15 & 58.4 \\
\hline $17 / 01 / 94$ & LA Wonderland & 95 & 6.8 & 0.2 & 5.9 & 9 & 110 \\
\hline $17 / 01 / 94$ & LA Wonderland & 185 & 6.8 & 0.3 & 4.17 & 7 & 169 \\
\hline $17 / 01 / 94$ & Antelope Buttes & 0 & 6.8 & 0.1 & 5.5 & 15 & 44.9 \\
\hline $17 / 01 / 94$ & Antelope Buttes & 90 & 6.8 & 0.1 & 3.42 & 14 & 67.0 \\
\hline $19 / 09 / 85$ & Filo de Caballo & NS & 8.1 & 0.2 & 2.86 & 25 & 67.2 \\
\hline $19 / 09 / 85$ & Filo de Caballo & EW & 8.1 & 0.2 & 3.0 & 31 & 69.2 \\
\hline $25 / 04 / 89$ & Los magueyes & EW & 6.9 & 0.1 & 3.9 & 9 & 7.34 \\
\hline $25 / 04 / 89$ & Los magueyes & NS & 6.9 & 0.1 & 3.0 & 8 & 7.25 \\
\hline $30 / 09 / 99$ & Mezcala & NS & 7.5 & 0.2 & 2.7 & 36 & 9.90 \\
\hline $30 / 09 / 99$ & Mezcala & EW & 7.5 & 0.2 & 4.4 & 37 & 12.3 \\
\hline
\end{tabular}

La figura 13 muestra los espectros de Fourier de los acelerogramas listados en la tabla 1, así como los espectros de los ruidos blancos equivalentes asociados a cada registro. Se observa claramente que todos los registros tienen un espectro de Fourier de banda ancha.

Los resultados obtenidos con el método propuesto se compararon con resultados calculados mediante dos reglas para estimar las demandas de $E_{H \mu}$ en osciladores, obtenidas de análisis estadísticos, desarrolladas por otros investigadores. Las reglas consideradas fueron:

a) Fajfar y Vidic (1992) para estimar la demanda de $E_{H \mu}$ en osciladores bilineales con diez por ciento de endurecimiento por deformación propusieron la siguiente expresión.

$$
E_{H \mu}=\left(\frac{\gamma \mu S A}{R_{\mu} \omega_{0}}\right)^{2}
$$

donde $S A$ es la seudoaceleración y $\gamma$ esta definido por: 


$$
\begin{aligned}
& \gamma=z_{T} z_{\mu} z_{g} \\
& z_{T}=\left\{\begin{array}{lll}
0.90 & \text { Si } \quad T \leq T_{1} \\
0.90-0.25 \frac{T-T_{1}}{T_{2}-T_{1}} & \text { Si } \quad T_{1} \leq T \leq T_{2} \\
0.65 & \text { Si } \quad T \geq T_{2}
\end{array}\right. \\
& z_{\mu}=\frac{(\mu-1)^{c_{\mu}}}{\mu} \\
& z_{g}=\left(\frac{\int a(t)^{2} d t}{A_{\max } P G V}\right)^{c_{g}} \\
& T_{1}=2 \pi \frac{c_{v} P G V}{c_{a} A_{\max }} \\
& T_{2}=2 \pi \frac{c_{d} P G D}{c_{v} P G V}
\end{aligned}
$$

Para osciladores bilineales $c_{g}=0.4, c_{m}=0.7$ y $c_{a}=c_{v}=2$ y $c_{d}=1.8$ y $P G V, P G D$ son la velocidad y desplazamiento máximos del terreno, respectivamente.

b) Manfredi (2001) con base en el estudio de osciladores sujetos a 122 acelerogramas propuso la siguiente expresión para estimar $E_{H \mu}$ :

$$
\begin{aligned}
& E_{H \mu}=\left(\mu_{c}-1\right) n_{e q}\left(\frac{S A}{\omega_{0}}\right)^{2}\left(\frac{1}{R_{\mu}}\right)^{2} \\
& n_{e q}=1+0.18\left(R_{\mu}-1\right)^{3 / 5} I_{D} \delta^{-1 / 6} \tau^{-1 / 2} \\
& I_{D}=\frac{\int_{0}^{d u r} a(t)^{2} d t}{A_{\max } P G V} \\
& \tau=\left\{\begin{array}{lll}
\frac{T}{T_{1}} & \text { Si } & T \leq T_{1} \\
1 & \text { Si } & T>T_{1}
\end{array}\right.
\end{aligned}
$$


$\mu_{c}$ es la demanda de ductilidad cíclica y para $\xi=0.05, \delta=1$.

Para facilitar la aplicación de la propuesta de Manfredi $\mu_{c}$ se aproximó en función de la demanda de ductilidad máxima $(\mu)$ conforme:

$$
\mu_{c}=\mu+1
$$

La ecuación (75) resulta en una buena estimación de la ductilidad cíclica si la curva fuerzadesplazamiento del oscilador tiende a permanecer simétrica respecto al origen.

Se calcularon los espectros de $E_{H \mu}$ para los movimientos considerados en la muestra y se compararon con los valores obtenidos con las dos propuestas mencionadas anteriormente y el procedimiento propuesto en este trabajo. Se consideraron valores de $T$ de 0.1 a 5 segundos y de $\mu$ de $1.5,2,3,4$ y 6 .

La comparación del nivel de exactitud entre las propuestas consideradas se realizó calculando el error logarítmico promedio para cada propuesta considerada, conforme a:

$$
\varepsilon_{\mathrm{ln}}=\sqrt{\frac{1}{n m p} \sum_{j=1}^{m} \sum_{i=1}^{n} \sum_{k=1}^{p}\left(\ln \left(\frac{E_{H \mu}}{\overline{E_{H \mu}}}\right)\right)^{2}}
$$

donde $n, m, p$ son el número de acelerogramas, el número de ductilidades y el número de periodos considerados y $\overline{E_{H \mu}}$ es el valor estimado de $E_{H \mu}$.

La figura 14 compara los errores logarítmicos promedio asociados a las diferentes propuestas consideradas para estimar $E_{H \mu}$ Como puede observarse, para los 16 registros considerados el procedimiento propuesto conduce al menor error logarítmico promedio, a pesar de que no se realizó ninguna regresión estadística para minimizar el error.

El error promedio mas grande se obtiene al utilizar la propuesta de Fajfar, aunque debe reconocerse que esta formulación fue desarrollada para sistemas con endurecimiento por deformación y aquí se aplicó a osciladores elastoplásticos perfectos.

El error promedio tiende a permanecer constante para la propuesta de Fajfar mientras que para la expresión de Manfredi y la desarrollada en este trabajo el nivel de error disminuye al aumentar la ductilidad, como se muestra en la figura 14. 


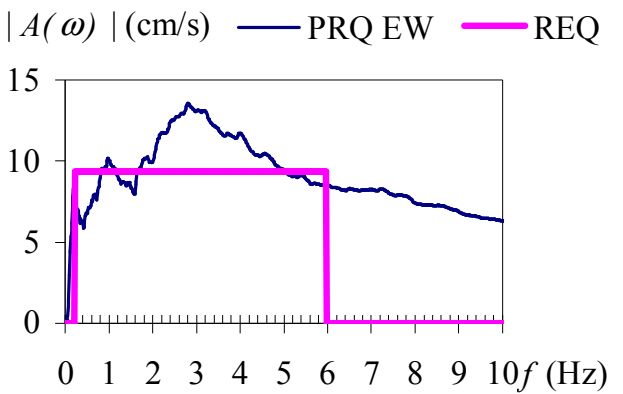

$|A(\omega)|(\mathrm{cm} / \mathrm{s})-$ PRQ NS - REQ
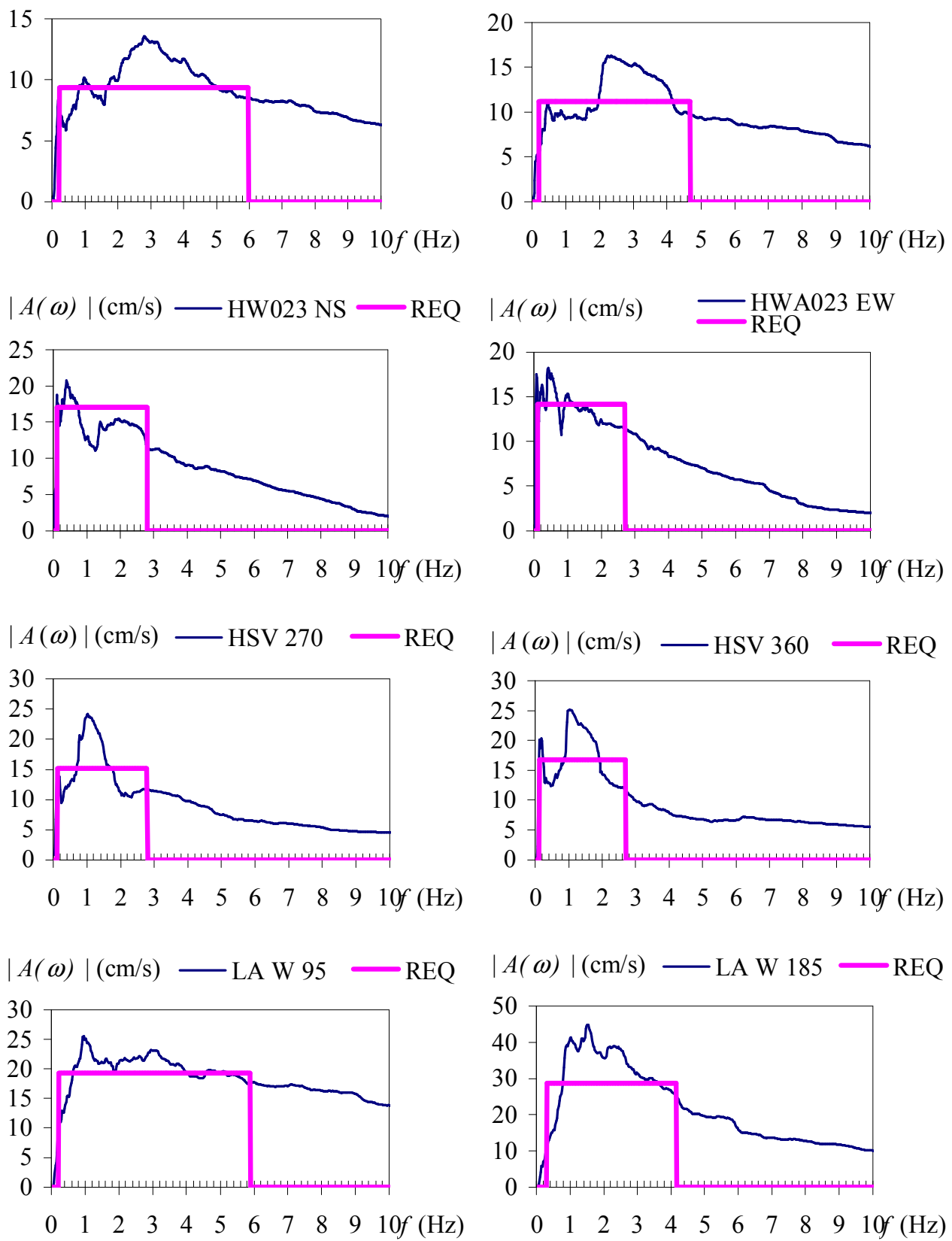

Figura 13. Espectros de amplitudes de Fourier de los acelerogramas utilizados y de los ruidos blancos equivalentes considerados.

Para el método presentado el error tiende a disminuir al aumentar la demanda ductilidad debido a que al disminuir la resistencia se incrementa el número de fluencias en el oscilador. $\mathrm{Al}$ aumentar el número de fluencias la respuesta del oscilador se asemeja más al proceso que dio origen a la solución propuesta. 
Para complementar estas observaciones la figura 15 muestra los errores logarítmicos, promedio como función del periodo, asociados a las diferentes propuestas consideradas.
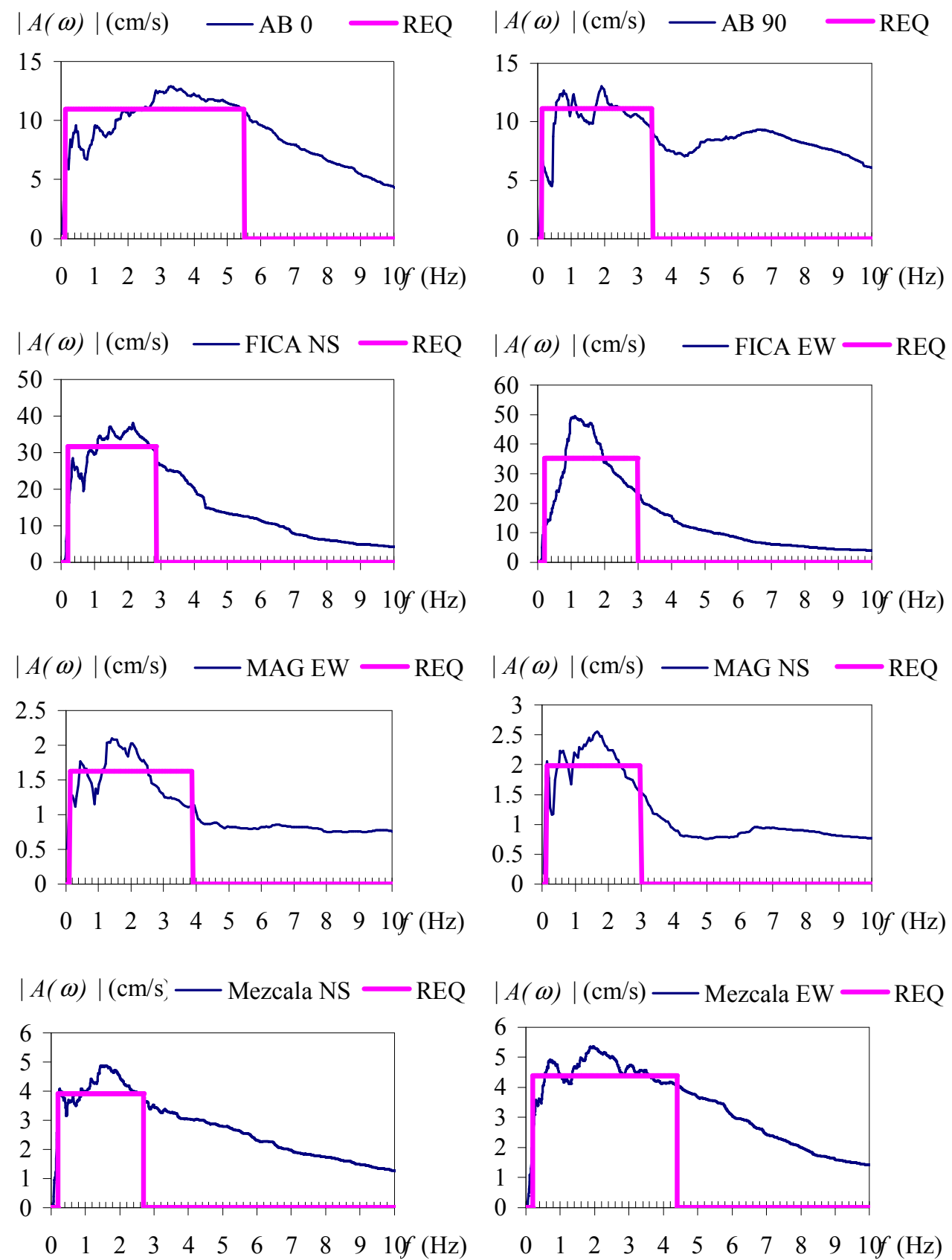

Figura 13. (Continua)

Se observa que a partir de un periodo de 0.5 segundos el error tiende a ser independiente del periodo para el método propuesto y para la ecuación de Manfredi, mientras que los errores asociados a la propuesta de Fajfar muestran una variación con el periodo. 

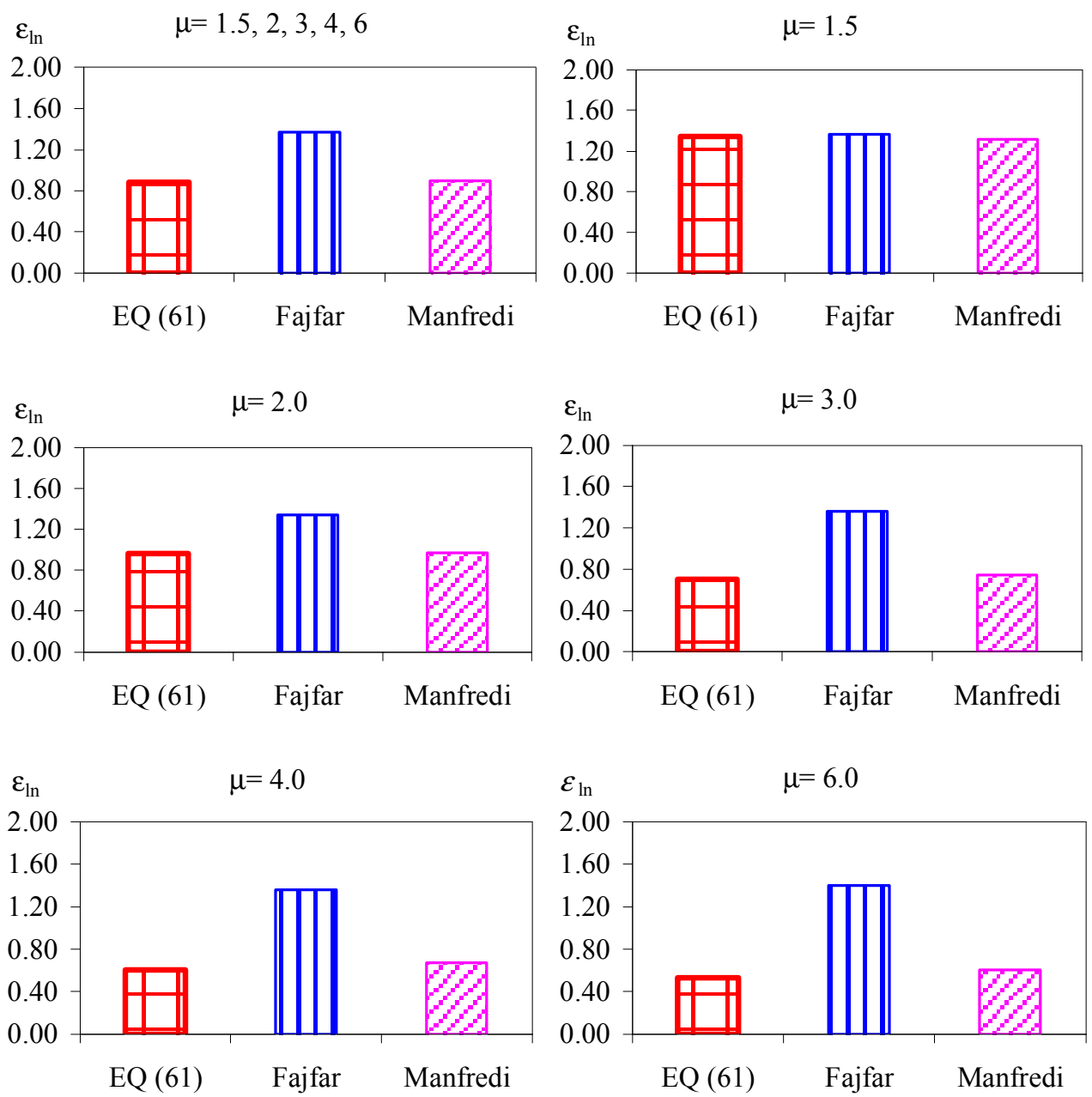

Figura 14. Errores logarítmicos promedio asociados a diferentes propuestas para estimar $E_{H \mu}$, $\xi=0.05$.

Para periodos menores a 0.5 segundos el error asociado al método propuesto tiende a aumentar en forma considerable. Esto es debido a que dichos periodos tienden a acercarse al límite de la banda útil de los acelerogramas de la muestra considerada.

Con base en lo mostrado en las figuras 14 y 15 puede concluirse que la propuesta desarrollada tiene un nivel de aproximación razonable en comparación con las expresiones obtenidas de análisis estadísticos disponibles.

Cabe mencionar que las propuestas Fajfar y Manfredi tienen la ventaja de que su aplicación práctica es mas sencilla que el método propuesto. Sin embargo, el método presentado requiere de menos información para su aplicación que las formulaciones de Fajfar y Manfredi. 
Como puede observarse en las ecuaciones 62 a 72 para aplicar las ecuaciones de Fajfar y Manfredi es necesario conocer un acelerograma. En cambio la aplicación de las expresiones presentadas no está necesariamente asociada a un acelerograma, lo cual permite estimar las demandas de energía histerética de un sitio determinado sólo en función del espectro de amplitudes de Fourier esperado, el cual puede estimarse mediante diferentes técnicas disponibles como leyes de atenuación y funciones de transferencia. Otra manera de ver lo anterior es que, como se muestra en este trabajo, toda la información necesaria para estimar la demanda de energía histerética de osciladores elastoplásticos sujetos a excitaciones sísmicas de banda ancha se encuentra contenida en el espectro de amplitudes de Fourier de la aceleración del suelo.

Es importante mencionar que el método presentado es aplicable sólo a osciladores elastoplásticos sujetos a movimientos de banda ancha, como los mostrados en la figura 13 .

Aspectos importantes a tratar en investigaciones futuras son: ampliar el caso de estudio a osciladores con otros comportamientos histeréticos, estudiar la aplicación a sistemas de varios grados de libertad y encontrar soluciones para otras formas de densidades espectrales de potencia.
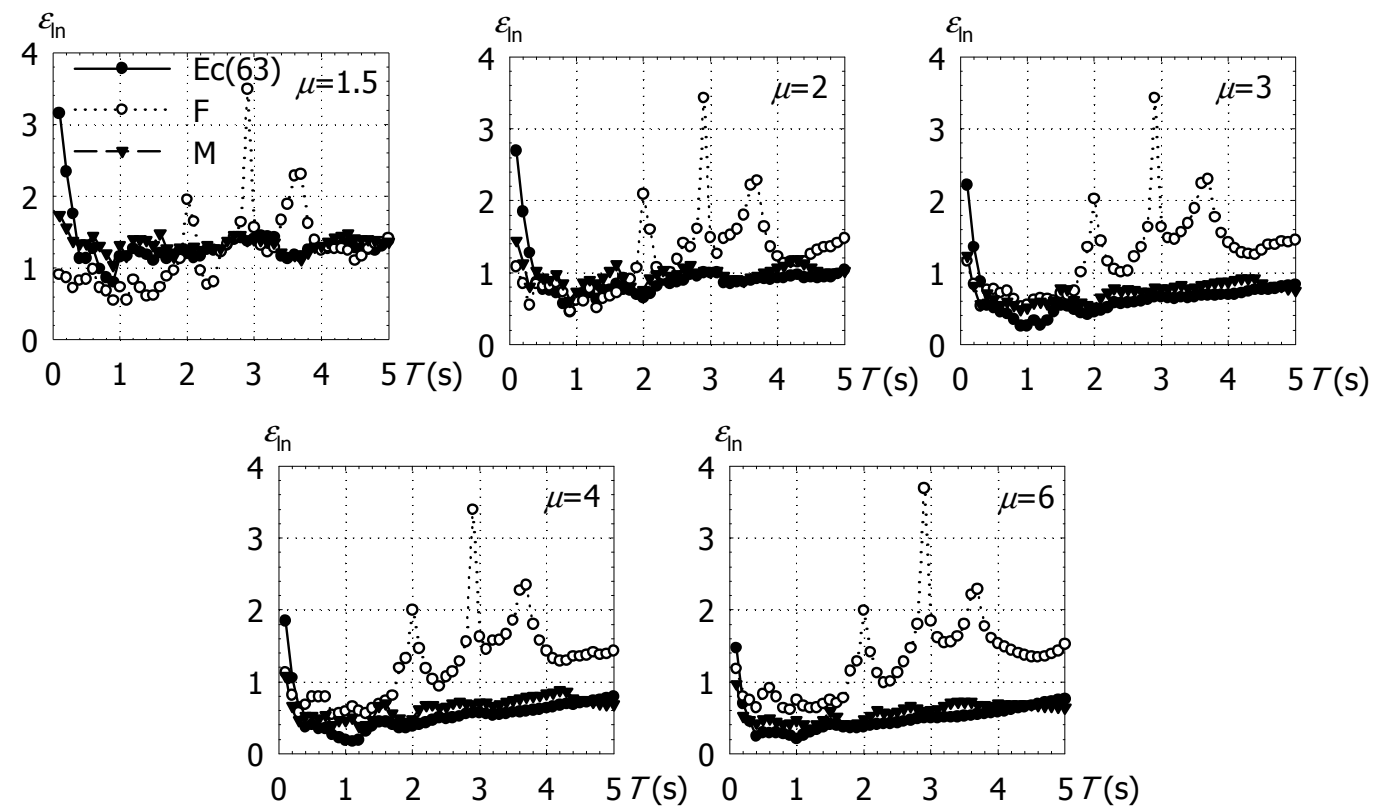

Figura 15. Errores logarítmicos promedio como función del periodo para diferentes estimaciones de $E_{H \mu}, \xi=0.05$

\section{CONCLUSIONES}

Se ha presentado una solución teórica para estimar las demandas de energía histerética disipada por osciladores elastoplásticos sujetos a ruido blanco gaussiano. Se ha mostrado que el procedimiento es aplicable a osciladores sujetos a excitaciones sísmicas de banda ancha y que el nivel de aproximación es similar a las propuestas existentes obtenidas de análisis estadísticos. 
Aunque en un contexto práctico las expresiones desarrolladas son difíciles de aplicar, el método tiene la ventaja, respecto a las propuestas obtenidas de estudios estadísticos, de que para estimar las demandas de energía histerética para un sitio determinado sólo se requiere conocer el espectro esperado de amplitudes de Fourier de la aceleración del suelo.

\section{AGRADECIMIENTOS}

Se agradecen las observaciones y comentarios realizados por los revisores anónimos de este artículo.

\section{REFERENCIAS}

Arroyo, D. y Terán, A. (2002), "Strength reductions factors to account for low cycle fatigue", Seventh US Conference on Earthquake Engineering, Boston, USA, CD.

Cooper G. R. y McGillem C. D. (1999), "Probabilistic methods of signal and systems analysis", Tercera edición, Oxford University Press.

Cosenza, E. y Manfredi, G. (1996), "Seismic design based on low cycle fatigue criteria", Memorias, XI Congreso Mundial de Ingeniería Sísmica, Acapulco, México, CD.

Fajfar, P. (1992), "Equivalent ductility factors taking into account low-cycle fatigue", Earthquake Engineering and Structural Dynamics, Vol. 21, pp. 837-848.

Fajfar, P y Vidic, T. (1994), "Consistent inelastic design spectra: hysteretic and input energy", Earthquake Engineering and Structural Dynamics, Vol. 23, pp. 523-537.

Iemura H. (1980) "Earthquake failure criteria of deteriorating hysteretic structures", VII World Conference on Earthquake engineering, Vol. 5, pp 81-88.

Karnopp, D. y Scharton, T. (1966), "Plastic deformation in random vibration", Journal of the acoustical society of America, Vol. 39, No. 6, pp 1154-1161.

Manfredi G. (2001), "Evaluation of seismic energy demand". Earthquake engineering and structural dynamics, Vol. 30, pp 485-499.

Mehanny, S. S. y Deierlein, G. G. (2000), "Modeling of assessment of seismic performance of seismic of composite frames with reinforced concrete columns and steel beams", Reporte No. 135, The John A. Blume Earthquake Engineering Center, Universidad de Stanford.

Roberts, J. B. (1968), "An approach to the first-passage problem in random vibration", Journal of Sound and Vibration, Vol. 8, No. 2, pp 301-328.

Park, Y. J. y Ang A. H. (1985), "Mechanistic seismic damage model for reinforced concrete", ASCE Journal of Structural Engineering, Vol. 111, No. 4, pp. $722-739$

Trifunac, M. D. y Brady, A. G. (1975). "A study on the duration of strong earthquake ground motion”, Bulletin of the Seimological Society of America, Vol. 65, No. 3, pp. 581-626. 
Terán, A. (1996), "Performance-based earthquake-resistant design of framed buildings using energy concepts", Tesis de Doctorado, Universidad de California, Berkeley

Terán, A. y Jirsa, J. O. (2003), "Un modelo simple para predecir la ocurrencia de fatiga de bajo número de ciclos", Memorias, XIV Congreso Nacional de Ingeniería Sísmica, León, México, CDROM.

Vanmarcke, E. H. y Lai, S. P. (1979), "Prediction of inelastic response spectra using random vibration", Proceedings of Engineering Mechanics, pp. 64-68.

Waterloo Maple Inc, (2002), "Maple 8.00"

Williams, M. S. y Sexsmith, R. G. (1995), "Seismic damage indices for concrete structures: a state of the art review”, Earthquake Spectra, Vol. 11, No. 2, pp. 319-349.

\section{ANEXO A. OBTENCIÓN DE LA ECUACIÓN PARA ESTIMAR E[ $\left.v^{2}\right]^{+}$}

La función de densidad de probabilidad de la velocidad del oscilador dado que se cruza la barrera en $X_{f}$ está definida por:

$$
p\left(v \mid \eta=X_{f}\right)=\frac{1}{\sqrt{2 \pi} S v c^{+}} e^{-\frac{1}{2} \frac{\left(v-E v c^{+}\right)^{2}}{\left(s v c^{+}\right)^{2}}}
$$

donde: $E v c^{+}$y $S v c^{+}$son el valor esperado y la desviación estándar condicionales de la velocidad cuando el desplazamiento es igual a $X_{f}$ y son definidos por las ecuaciones 37 y 38 , respectivamente.

Partiendo de la ecuación A1 es posible obtener la densidad de probabilidad condicional de la velocidad dado que el desplazamiento es igual al desplazamiento de fluencia y que el oscilador tiene velocidad positiva $\left(p\left(v \mid \eta=X_{f} \cap v>0\right)\right)$. Para ello, se trabaja con las siguientes funciones de distribución de probabilidades (Cooper y McGillem, 1999).

$$
P\left(\nu \mid \eta=X_{f} \cap v>0\right)= \begin{cases}0 & \text { Si } \quad v<0 \\ \frac{\int_{0}^{v} p\left(v \mid \eta=X_{f}\right) d v}{1-\int_{-\infty}^{0} p\left(v \mid \eta=X_{f}\right) d v} & \text { Si } \quad v \geq 0\end{cases}
$$

Una vez conocida la función de distribución de probabilidades, la función de densidad se obtiene derivando a la función de distribución con respecto a la velocidad. 
$p\left(v \mid \eta=X_{f} \cap v>0\right)=\frac{d}{d v} P\left(v \mid \eta=X_{f} \cap v>0\right)$

Realizando las operaciones se obtiene:

$p\left(v \mid \eta=X_{f} \cap v>0\right)= \begin{cases}0 & S i \quad v<0 \\ \left(\frac{1}{\sqrt{2 \pi} S v c^{+}} e^{-\frac{1}{2} \frac{\left(v-E v c^{+}\right)^{2}}{\left(S v c^{+}\right)^{2}}}\right) \frac{1}{\Phi\left(\frac{E v c^{+}}{S v c^{+}}\right)} & \text {Si } \quad v \geq 0\end{cases}$

Finalmente, el valor de $\mathrm{E}\left[v^{2}\right]^{+}$se obtiene conforme a:

$$
\begin{aligned}
& E\left[v^{2}\right]^{+}=\int_{0}^{\infty} v^{2} p\left(v \mid \eta=X_{f} \cap v>0\right) d v \\
& E\left[v^{2}\right]^{+}=\left(E v c^{+}\right)^{2}+\left(S v c^{+}\right)^{2}+\frac{E v c^{+} S v c^{+}}{\sqrt{2 \pi} \Phi\left(\frac{E v c^{+}}{S v c^{+}}\right)} e^{-\frac{1}{2}\left(\frac{E v c^{+}}{S v c^{+}}\right)^{2}}
\end{aligned}
$$

